\title{
Development of injectable and durable kefiran hydro-alcoholic gels
}

Maria Antonietta Sabatino ${ }^{1}$, Francesco Carfi Pavia ${ }^{1,2}$, Salvatrice Rigogliuso ${ }^{3}$, Daniela Giacomazza ${ }^{4}$, Giulio Ghersi ${ }^{5}$, Vincenzo La Carrubba ${ }^{1,6}$, Clelia Dispenza $^{1,4, *}$

${ }^{1}$ Dipartimento di Ingegneria, Università degli Studi di Palermo, Viale delle Scienze Ed.6, 90128 Palermo, Italy.

${ }^{2}$ ATeN Center, Università degli Studi di Palermo, Viale delle Scienze ed. 18, 90128 Palermo, Italy

${ }^{3}$ Abiel S.r.l (Applicazioni Biomediche e Industriali di Enzimi Litici) presso STEBICEF, Università degli Studi di Palermo, Viale delle Scienze Ed. 16, 90128 Palermo, Italy.

${ }^{4}$ Istituto di BioFisica (IBF), Consiglio Nazionale delle Ricerche, Via U. La Malfa, 153, 90146 Palermo (PA), Italy

${ }^{5}$ Dipartimento di Scienze e Tecnologie Biologiche Chimiche e Farmaceutiche, Università degli Studi di Palermo, Viale delle Scienze Ed. 16, 90128 Palermo, Italy.

${ }^{6}$ Consorzio Interuniversitario per la Scienza e Tecnologia dei Materiali (INSTM), Unità di Ricerca di Palermo, Viale delle Scienze ed. 6, 90128 Palermo, Italy.

\section{* Corresponding author:}

Clelia Dispenza: Dipartimento di Ingegneria. Università degli Studi di Palermo. Viale delle Scienze 6.90128 Palermo, Italy. Email: clelia.dispenza@unipa.it 


\section{ABSTRACT}

Injectable, in-situ forming kefiran gels have been developed for potential applications as implantable drug delivery devices or scaffolds for tissue regeneration. Concentrated solutions $(4,5$ and $6 \% \mathrm{w})$ of kefiran, extracted from kefir grains, have been assessed in term of viscosity and injectability through G26 syringe needles, and for their ability to undergo gelation upon mixing with different alcohols. Propylene glycol (PG) has been selected as gelling agent because it ensures homogenous gelation in relatively short times (from few minutes up to 6 hours). The investigation of the rheological behavior of kefiran/PG gels varying polymer concentration and temperature $\left(25^{\circ} \mathrm{C}\right.$ and $\left.37^{\circ} \mathrm{C}\right)$ has provided interesting hints to support a possible gelation mechanism that accounts also for the observed influence of the alcohol type. Finally, the study of kefiran/PG gels has been complemented with the investigation on selected formulations of the swelling/degradation behavior upon immersion in isotonic buffer solution for up to 40 days at $37^{\circ} \mathrm{C}$; of the ability of the gels to retain and/or release two model molecules; and with in vitro cell viability and cytotoxicity tests, to support the absence of toxic effects on cells induced by direct contact with the gels or by leached components from these gels.

\section{KEYWORDS}

Kefiran gels; in-situ forming gels; gelation. 


\section{INTRODUCTION}

Hydrogels are a class of materials that for their soft and elastic consistency and high water content closely mimic the properties of soft biological tissues. For this reason, they are widely investigated as scaffolds in regenerative medicine and in-situ forming implants for drug delivery. ${ }^{1-3}$

They are characterised by an internal porous structure, permeable to biological fluids and molecular oxygen, and by hydrophobic pockets and functional groups that can host or bind proteins with a therapeutic function or with the ability to stimulate host cells migration, proliferation and/or differentiation toward a desired cell phenotype. For long-term applications, the hydrogel matrix may also ensure the stability and hence the activity of the incorporated bioactive factors to maximize the resulting benefits..$^{4-6}$ When hydrogels are fully or partially biodegradable, erosion and/or degradation kinetics can be exploited for temporal modulation of drug release or to balance cell remodeling rate and new tissue formation..$^{7,8}$

Formulations undergoing in situ sol-gel transition are suitable candidates to reach deep compartments of the human body and/or to fill large body cavities with complex shapes in minimally invasive interventions..$^{9-11}$ For the development of injectable, in-situ forming gels stringent requirements need to be met: (i) all components of the formulation, their eventual reaction or degradation products need to be biocompatible; (ii) the viscosity of the precursor solutions should be sufficiently low to allow a homogeneous dispersion of drugs/cells/bioactive factors before gelation, easy extrusion through small gauge syringe and minimal residual mass in the syringe; (iii) the gelation conditions and rates should guarantee the formation of homogeneous network and avoid uncontrolled diffusion of the injected material away from the intended locus of injection towards the surrounding tissues, or cause tissues overheating and/or damage..$^{12-14}$

In situ-forming gels can be obtained by inducing the sol-gel transition of polymers that are responsive to changes in temperature ${ }^{6,15-17}$ or $\mathrm{pH}^{18}$ addition of specific ions ${ }^{19}$ or co-solutes; ${ }^{20-22}$ and/or by promoting covalent crosslinking between different chains through chemical reactions occurring in physiological conditions..$^{23}$ 
Both synthetic and natural source polymers have been used for the purpose, including several polysaccharides such as hyaluronic acid, chitosan, alginate, chondroitin sulfate, agarose, native and degalactosilated xyloglucan, gellan gum; and polyaminoacids such as collagen, gelatin, fibrin and silk..$^{6,24-26}$

Kefiran, obtained by Lactobacillus kefiranofaciens, is a branched exopolysaccharide, constituted by D-glucose (Glc) and D-galactose (Gal) with almost 1:1 molar ratio. The backbone is composed by (1 $\rightarrow$ 6)-linked Glc, $(1 \rightarrow 3)$-linked Gal, $(1 \rightarrow 4)$-linked Gal, $(1 \rightarrow 4)$-linked Glc and $(1 \rightarrow 2,6)$-linked Gal, with Glc branches attached to the O-2 of the Gal residues. A Glc residue terminates the chain backbone. ${ }^{27,28}$ In the solid state, kefiran is a semi-crystalline polymer with an estimated degree of crystallinity of ca. $30 \% .29,30$

Kefir and kefiran have been used in food science and technology for their antibacterial, antifungal, antioxidant and anti-inflammatory properties; for their ability to reduce serum cholesterol levels and strengthening the immune system; ${ }^{31-33}$ and to produce biodegradable edible films. ${ }^{34}$ More recently, a few biomedical applications of kefiran aqueous solutions have been proposed. ${ }^{35-38}$

Only few studies report about kefiran gelation. Generally, kefiran gels are formed by freeze-thaw cycles of diluted or semi-diluted (0.5-2 \%w) polymer aqueous solutions, ${ }^{39}$ also in the presence of significant amounts $(2-50 \% \mathrm{w})$ of sugar molecules; ${ }^{40}$ or by mixing with whey proteins ${ }^{41}$ Mukai et al. describe gels formed by mixing kefiran aqueous solutions with either ethanol or ethylene glycol and storage for $24 \mathrm{~h}$ at $5^{\circ} \mathrm{C} .{ }^{42}$ Gelation conditions that imply low temperature treatments or require high viscosity formulations are not applicable for the development of in-situ forming scaffolds or implants. In this paper, the use of kefiran for the development of a new family of injectable, in-situ forming gels, characterized by fast gelation in physiological conditions and slow erosion kinetics is demonstrated.

Kefiran was extracted by commercial kefir grains. Samples of the purified polymer were subjected to hydrolytic digestion followed by ninhydrin test to assess the presence of protein residues and by high performance chromatography analysis (HPLC) to quantify the constituent monosaccharides. The 
polymer was also characterized by thermo-gravimetric analysis (TGA), static light scattering (SLS) and gel filtration chromatography (GFC).

Kefiran aqueous solutions at 4,5 and $6 \% \mathrm{w}$ were characterized for their rotational viscosity and injectability. Four different alcohols, ethanol, 2-(2-ethoxyethoxy)ethanol, propylene glycol, glycerol, were screened as gelling agents of the three above solutions. The pre-selection of these alcohols and their amount in the formulation $(20 \% \mathrm{w})$ was made taking into account the limitations in terms of type and maximum concentration of alcohol that can be used for subcutaneous or intramuscular injectable formulations ${ }^{43}$ From a first screening among the alcohols, based on visual inspection of the time to gel and homogeneity of the formed gels, propylene glycol was selected as gelling agent. A further refinement of the selection was performed among the three kefiran/propylene glycol systems obtained from the three kefiran aqueous solutions based on their dynamic rheological behavior. Considering the effect of the different alcohols on the gelation behavior of kefiran solutions and the influence of polymer concentration and temperature on the rheological behavior of the kefiran/propylene glycol systems, a mechanism of gelation can be proposed. The hydrolytic degradation behavior of kefiran/propylene glycol gels in isotonic buffer solution at $37^{\circ} \mathrm{C}$ for up to 40 days, their chemical and morphological evolution resulting from the prolonged incubation, was also investigated. Finally, release experiments using two model molecules as well as qualitative and quantitative cytotoxicity tests were performed.

\section{MATERIALS AND METHODS}

\subsection{Materials}

Kefir grains were purchased from Kefiring, shipped under refrigerated temperature $\left(4^{\circ} \mathrm{C}\right)$ to maintain the grains inactive. Ethanol $(\mathrm{EtOH}), 2-(2-$ ethoxyethoxy)ethanol (EEE), propylene glycol (PG), glycerol (Gro), $\mathrm{NaCl}, \mathrm{KH}_{2} \mathrm{PO}_{4}, \mathrm{Na}_{2} \mathrm{HPO}_{4}$, pullulan standards, fluorescein isothiocynate (FITC)labelled insulin, Atto $633^{\circledR}$ amine, serum bovin albumin (BSA), L-glutamine, ninhydrin, $\mathrm{SnCl}_{2}, 2$ - 
methoxyethanol (MeOEt), citrate buffer, $\mathrm{HCl}$ and trifluoroacetic acid (TFA) were purchased from Sigma-Aldrich and employed as received. MilliQ water was used for all preparations.

\subsection{Isolation and purification of kefiran}

Kefiran extraction was performed by following the Piermaria et al. procedure, with slight modifications.$^{39,40,44}$ A weighed amount of kefir grains $(1-10 \mathrm{wt} \%)$ was placed in hot water $\left(90^{\circ} \mathrm{C}\right)$ for $120 \mathrm{~min}$. Then, the mixture was centrifuged (Thermo Scientific SL40R Centrifuge) at 10,000 $g$ for $20 \mathrm{~min}$ at $20^{\circ} \mathrm{C}$ to remove bacteria and proteins. The supernatant was recovered and the polysaccharide precipitated by addition of $\mathrm{EtOH}$ in a $1: 1$ volume ratio and left at $-20^{\circ} \mathrm{C}$ overnight. The dispersion was centrifuged again at $10,000 \mathrm{~g}$ for $20 \mathrm{~min}$ at $4{ }^{\circ} \mathrm{C}$. The solubilisation and precipitation procedure was repeated twice. Finally, the precipitate was lyophilized. In the following, the purified solid polymer will be named "kefiran".

\subsection{Characterization of chemical structure and purity of extracted kefiran}

The presence of residual proteins in kefiran was quantified using the ninhydrin assay. ${ }^{45}$ In particular, $10 \mathrm{mg}$ of polymer were hydrolyzed in microfuge tubes at $100^{\circ} \mathrm{C}$ for $24 \mathrm{~h}$ by adding $500 \mu \mathrm{l}$ of $6 \mathrm{M}$ $\mathrm{HCl}$. The residue obtained after evaporating to dryness was dissolved in $1 \mathrm{ml}$ of water. The same treatment was applied to BSA and L-Glutamine used as standards. Ninhydrin and $\mathrm{SnCl}_{2}$ solutions were prepared immediately before use from $200 \mathrm{mg}$ of ninhydrin in $5 \mathrm{ml}$ of MeOEt and $5 \mathrm{ml}$ of citrate buffer $\left(0.2 \mathrm{M}\right.$ at pH 5), and from $32 \mathrm{mg}$ of $\mathrm{SnCl}_{2}$ in $2 \mathrm{ml}$ of $0.1 \mathrm{M} \mathrm{HCl}$, respectively. $150 \mu$ l of kefiran solution was mixed with $600 \mu \mathrm{l}$ of ninhydrin solution and $30 \mu 1 \mathrm{SnCl}_{2}$ solution. The reaction was carried out at $100{ }^{\circ} \mathrm{C}$ for $15 \mathrm{~min}$. Samples were cooled in an ice water bath to stop the reaction. 200 $\mu 1$ of each sample was mixed with $1 \mathrm{ml}$ of $50 \%(\mathrm{v} / \mathrm{v})$ propanol/distilled water and vortexed. The UVvis absorption of these solutions was read on a plate reader spectrophotometer at the wavelength of $565 \mathrm{~nm}$. The same protocol was applied to the protein standards. 
The quantitative analysis of monosaccharides composing the biopolymer was performed by hydrolytic digestion and HPLC analysis. More in detail, kefiran (10 mg) was hydrolyzed with TFA $(5 \mathrm{ml}, 2 \mathrm{M})$ at $90^{\circ} \mathrm{C}$ for $5 \mathrm{~h}$. Then, the solution was cooled and evaporated with vacuum rotovapor to dryness. EtOH was added and evaporated three times to remove trifluoroacetic acid. The quantity of monosaccharides was determined using an Agilent 1200 HPLC apparatus with a refractive index detector and Dionex Carbopac PA1 column, using $\mathrm{NaOH}(16 \mathrm{mM})$ as eluent at a flow rate of 0.9 $\mathrm{mL} / \mathrm{min}$ at $25^{\circ} \mathrm{C}$. Samples for HPLC were prepared in the elution solvent at $0.1 \% \mathrm{w}$. For quantitative analysis, calibration curves of glucose or galactose were built.

Thermal-gravimetric analysis (TGA) was conducted with a LabsysEVO TGDTA/DSC (Setaram, France). Samples of kefiran $(10 \mathrm{mg})$ were pre-conditioned at $25^{\circ} \mathrm{C}$ for $10 \mathrm{~min}$, and tested in the temperature range of $20-500{ }^{\circ} \mathrm{C}$ at a heating rate of $7^{\circ} \mathrm{C} / \mathrm{min}$. The characteristic temperatures associated to the various phenomena are calculated as the peak temperature of the first derivative function (DTG).

\subsection{Determination of the molecular properties of the extracted kefiran}

Weight average molecular weight $\left(\mathrm{M}_{\mathrm{w}}\right)$, second virial coefficient $\left(\mathrm{A}_{2}\right)$ and radius of gyration $\left(\mathrm{R}_{g}\right)$ of kefiran were estimated from multi-angle static light scattering measurements performed using a Brookhaven BI-9000 correlator and a $50 \mathrm{~mW}$ Melles Griot He-Ne laser tuned at $632.8 \mathrm{~nm}$ at $20 \pm$ $0.1^{\circ} \mathrm{C}$. The refractive index increment, $\mathrm{dn} / \mathrm{dc}=0.143$ of kefiran in aqueous solution, was measured by using a Brookhaven Instruments differential refractometer at the wavelength of $620 \mathrm{~nm}$. Measurements were carried out on kefiran aqueous solutions at five concentrations, in the range 0.02 $\% \mathrm{w}$ to $0.2 \% \mathrm{w}$, after filtration with $0.45 \mu \mathrm{m}$ cellulose acetate syringe filters (Millipore). Each measurement was repeated at least three times on samples produced from different batches. Mw, $\mathrm{A}_{2}$ and $\mathrm{R}_{g}$ were determined via Zimm plot analysis. ${ }^{46}$

Gel filtration chromatography was conducted using a Shodex SB HQ (804 and 806) columns coupled with an Agilent 1260 Infinity HPLC equipped with a refractive index detector. All systems were 
diluted with water to the final concentration of $0.1 \% \mathrm{w}$. Samples were eluted with a flux of $0.6 \mathrm{ml} / \mathrm{min}$ of $0.02 \% \mathrm{w}$ sodium azide aqueous solution. Prior to injection in the column, the polymer aqueous solutions were filtered with 1.2, 0.8. 0.45 and $0.22 \mu \mathrm{m}$ cellulose acetate (Millipore) syringe filters. Each measurement was repeated three times on samples produced from different batches. Chromatograms of the samples were compared with the chromatograms of pullulan standard solutions.

\subsection{Shear viscosity measurements and injectability assessment}

Kefiran aqueous solutions at 4,5 and $6 \% \mathrm{w}$ were prepared by magnetic stirring for $2 \mathrm{~h}$ at $70^{\circ} \mathrm{C}$. Solutions were named as $\operatorname{Kef}(\mathrm{X})$, where $\mathrm{X}$ is the polymer concentration.

Shear viscosity measurements were performed on $\operatorname{Kef}(4), \operatorname{Kef}(5)$ and $\operatorname{Kef}(6)$ using a stress-controlled Rheometer AR-1000 (TA Instruments) equipped with an acrylic cone-plate geometry (angle $<0.0174$ $\operatorname{rad}$, radius $20 \mathrm{~mm}$, truncation $30 \mu \mathrm{m})$ at $25^{\circ} \mathrm{C}$.

The same solutions were injected at room temperature through a $1 \mathrm{ml}$ TERUMO Europe N.V. syringe with a G26 x 1/2" needle. Injection time and residual mass of material in the syringe were measured.

\subsection{Preparation of kefiran hydrogels}

Gelled samples were obtained by mixing at room temperature $\operatorname{Kef}(4), \operatorname{Kef}(5)$ and $\operatorname{Kef}(6)$ with each of the four alcohols at 4:1 mass ratio. The final polymer concentration in the gels is $3.2,4.0$ and 4.8 $\% \mathrm{w}$, respectively. The various gel systems are named with the code of the solution used and the acronym of the alcohol (e.g. $\operatorname{Kef}(\mathrm{X}) / \mathrm{PG})$.

\subsection{Visual inspection and tilting test of kefiran/alcohol hydrogels}

$\operatorname{Kef}(4), \operatorname{Kef}(5)$ and $\operatorname{Kef}(6)$ solutions mixed with the various alcohols were visually inspected soon after preparation and at regular intervals for up to $48 \mathrm{~h}$ to check homogeneity and occurrence of syneresis. 
A "tilting test" ${ }^{47}$ was also performed by mixing polymer solution with the alcohol within transparent cylindrical tubes (12 mm diameter). The tubes were tilted at various regular time intervals to assess if the material was able to flow.

\subsection{Dynamic mechanical rheological analysis}

Rheological measurements were performed with a controlled stress Rheometer (AR-1000, TA Instruments, UK) equipped with an acrylic plate-plate geometry (radius $20 \mathrm{~mm}$, gap $1000 \mu \mathrm{m}$ ). The temperature was controlled by a built-in Peltier system set at $25 \pm 0.1^{\circ} \mathrm{C}$ or at $37 \pm 0.1^{\circ} \mathrm{C}$. Preliminary "strain sweep" tests were carried out on $\operatorname{Kef}(4) / \mathrm{PG}, \operatorname{Kef}(5) / \mathrm{PG}$ and $\operatorname{Kef}(6) / \mathrm{PG}$ soon after mixing $(\mathrm{t}=0)$; after $2 \mathrm{~h}$ and $48 \mathrm{~h}$ of incubation at $25 \pm 0.1^{\circ} \mathrm{C}$ and on $\operatorname{Kef}(6) / \mathrm{PG}$ after $2 \mathrm{~h}$ and $48 \mathrm{~h}$ incubation at $37 \pm 0.1^{\circ} \mathrm{C}$. It was observed that the strain amplitude of $5 \times 10^{-3}$ was well within the linear viscoelastic region for all the above systems and it was selected as the constant strain amplitude for all the “frequency sweep" measurements performed.

Gelation kinetics of $\operatorname{Kef}(4) / \mathrm{PG}, \operatorname{Kef}(5) / \mathrm{PG}$ and $\operatorname{Kef}(6) / \mathrm{PG}$ systems were studied by a series of consecutive frequency sweeps from $0.05 \mathrm{~Hz}$ to $50 \mathrm{~Hz}$ (time needed per sweep: $15 \mathrm{~min}$ ) over $24 \mathrm{~h}$ at $25 \pm 0.1^{\circ} \mathrm{C}$. Samples were mixed in small beakers and immediately transferred onto the rheometer plate.

Frequency sweeps tests were carried out on $\operatorname{Kef}(4), \operatorname{Kef}(5)$ and $\operatorname{Kef}(6)$ solutions and $\operatorname{Kef}(4) / P G$, $\operatorname{Kef}(5) / P G$ and $\operatorname{Kef}(6) / P G$ gels. Gel disks of the same shape and size of the rheometer plate (thickness of ca. $1000 \mu \mathrm{m}$ ) were prepared on Teflon molds and were either immediately tested; or incubated in closed glass boxes at room temperature for $48 \mathrm{~h}$ and then tested. Samples of $\operatorname{Kef}(6) / \mathrm{PG}$ were also incubated at $25 \pm 0.1{ }^{\circ} \mathrm{C}$ and $37 \pm 0.1^{\circ} \mathrm{C}$ for $2 \mathrm{~h}, 6 \mathrm{~h}, 12 \mathrm{~h}, 24 \mathrm{~h} 36 \mathrm{~h}$ and $48 \mathrm{~h}$.

For all type of measurements, the thin air/sample interface was coated with silicon oil to avoid water evaporation. Each measurement was repeated at least three times on samples produced from different batches. No significant differences were observed. 


\subsection{Hydrolytic degradation behaviour}

Ten cylindrical samples (diameter of ca. $6 \mathrm{~mm}$, height of ca. $6 \mathrm{~mm}$ ) of $\operatorname{Kef}(5) / \mathrm{PG}$ and $\operatorname{Kef}(6) / \mathrm{PG}$ were preconditioned at room temperature for $48 \mathrm{~h}$, then weighed and immersed in a large volume of isotonic PBS at $\mathrm{pH} 7.4$ and $37 \pm 0.1{ }^{\circ} \mathrm{C}$ for up to 40 days. The cylinders were weighed at predetermined time intervals, after blotting with filter paper. The weight change $(\Delta \mathrm{W} \%)$ was calculated as:

$$
\Delta \mathrm{W} \%=\frac{\left(W_{t}-W_{i}\right)}{W_{i}} \times 100
$$

where, $\mathrm{W}_{\mathrm{t}}$ corresponds to the weight of the sample at incubation time $\mathrm{t}$, and $\mathrm{W}_{\mathrm{i}}$ represents the initial weight. Systems immersed in isotonic PBS for 40 days at $37^{\circ} \mathrm{C}$ are named after "aged".

The occurrence of eventual modification of kefiran structure upon incubation was investigated by Fourier-Transform Infrared (FITR) analysis, performed using a FTIR Spectrometer (Spectrum Two, Perkin Elmer). Freeze dried samples of Kef(5)/PG and Kef(6) gels, just prepared and "aged”, were mixed with potassium bromide and compressed into a transparent pellet. Spectra were collected by accumulation of 32 scans between 4000 and $450 \mathrm{~cm}^{-1}$, with a resolution of $4 \mathrm{~cm}^{-1}$. A sample of kefiran was also analysed as reference.

The morphology of the freeze dried gel samples before and after degradation was inspected by Scanning Electron Microscopy (SEM) using a Versa 3D (FEI-Thermo Fischer, The Netherlands). Gels were repeatedly washed with water to extract PG and/or PBS salts (for the aged samples), freezedried, and gold coated by JFC-1300 gold coater (JEOL) for $90 \mathrm{~s}$ at $30 \mathrm{~mA}$ before scanning.

\subsection{Model molecules release experiments}

The water-soluble fluorescent probe, Atto $633\left(\lambda_{\text {abs } \max }=629 \mathrm{~nm}\right)$, and FITC-labelled insulin (FITC_Ins) $\left(\lambda_{\text {abs } \max }=490 \mathrm{~nm}\right)$ were loaded on the gels by adding known volumes $(100 \mu \mathrm{l})$ of Atto 
$633(0.1 \mathrm{~g} / \mathrm{L})$ or FITC_Ins $(1 \mathrm{~g} / \mathrm{L})$ to $4 \mathrm{~g}$ of $\operatorname{Kef}(5)$ or $\operatorname{Kef}(6)$ prior to mixing with PG. The loaded amounts of Atto 633 and FITC_Ins were $2.5 \mu \mathrm{g} / \mathrm{g}$ and $25 \mu \mathrm{g} / \mathrm{g}$ of gel, respectively.

Five samples of Atto 633- or FTIC_Ins-loaded gels (120 mg per sample, preconditioned for 48h at room temperature) were immersed in $5 \mathrm{ml}$ of fresh PBS medium kept under gentle stirring for $48 \mathrm{~h}$. At predetermined times, aliquots of the solutions were taken to be analysed through UV-vis absorption spectrophotometry (Jasco V-670 spectrophotometer, scan-speed $100 \mathrm{~nm} / \mathrm{min}$, band width $1 \mathrm{~nm}$, at room temperature) and UV-vis fluorescence spectrophotometry (JASCO FP-6500 spectrofluorimeter equipped with a Xenon lamp) and replaced with fresh PBS medium. The quantitative determination of Atto 633 released was performed by building a UV-vis absorption calibration curve.

\subsection{Cell viability and cytotoxicity assays}

In order to evaluate cell viability in the presence and in the absence of the $\operatorname{Kef}(6) / \mathrm{PG}$ gel, preosteoblastic MC3T3-E1 cells (Sigma Aldrich) were seeded at a density of 1x104/well into 24-well culture plate and cultured in 2D conditions in Dulbecco's modified Eagle's medium (DMEM, Sigma Aldrich), supplemented with $10 \%$ foetal bovine serum, $1 \%$ glutamine and $1 \%$ streptomycin/penicillin. Samples of about $100 \mathrm{mg}$ of gels were extensively equilibrated in DMEM and placed directly onto cells growing in complete DMEM medium. During incubation, leachable chemicals present in the gel can diffuse into the culture medium and contact the cell layer. Cell viability was evaluated as fluorescence intensity $\left(\lambda_{\mathrm{abs}}=530 \mathrm{~nm}, \lambda_{\mathrm{em}}=590 \mathrm{~nm}\right)$ at different times, up to 12 days, using AlamarBlue Cell Viability Reagent (Invitrogen).

Pre-osteoblastic MC3T3-E1 cells, seeded at a density of $1 \times 10^{4}$ on a cover glass placed inside a 24well culture plate, were grown in presence of $\operatorname{Kef}(6) / \mathrm{PG}$ gel in the same conditions of the viability assay described above, were fixed with $3.7 \%$ formaldehyde (Sigma) in complete PBS (Phosphate Buffer Saline with calcium and magnesium; EuroClone) after 2, 4 and 8 days of culture. Cells were stained using a mix of Phalloidine ${ }^{\mathrm{FITC}}$ (Sigma) for cytoskeleton identification (1:500) and DAPI 
(Sigma) for nucleus identification (1:20000) in PBS. The incubation with probes was carried out at $37^{\circ} \mathrm{C}$ for 15 minutes. Cover glass with cells were mounted and observed using an inverted Fluorescence Microscope (Leica).

Pre-osteoblastic MC3T3-E1 cells, seeded at concentration of $1 \times 10^{4}$ on a cover glass placed inside the 24-well culture plate, were grown (i) in complete DMEM medium (negative control); (ii) in a solution of Doxorubicin $100 \mu \mathrm{M}$, final concentration, added in the complete DMEM medium and incubated for two hours (positive control); and (iii) in complete DMEM medium with added $100 \mathrm{mg} / \mathrm{well}$ of Kef(6)/PG gel. At various incubation times samples were fixed with $3.7 \%$ formaldehyde (Sigma) in complete PBS and stained with Acridine Orange solution (Sigma) $[100 \mu \mathrm{g} / \mathrm{ml}]$ for $30 \mathrm{~s}$. Cover glass with cells were mounted and observed using an inverted Fluorescence Microscope. Acridine Orange is a metachromatic dye that can be used to observe apoptosis phenomenon caused on cells by cytotoxic agents. All experiments were carried out three times.

\section{RESULTS AND DISCUSSION}

\subsection{Chemical composition and structural characterisation of extracted kefiran}

Kefiran extracted by commercial kefir grains was subjected to hydrolytic digestion followed by (i) ninhydrin test to determine the content of residual proteins and (ii) HPLC to quantify its constituent monosaccharides. The total content of proteins resulted lower than $1.5 \% \mathrm{w}$, confirming the efficacy of the purification procedure. The molar ratio between Gal and Glc was 1:1.1, value that is in good agreement with literature data. ${ }^{27,28}$

The weight average molecular weight, the radius of gyration and the 2 nd virial coefficient of the extracted and purified biopolymer were determined by Zimm plot analysis of SLS data collected for dilute aqueous solutions (see Figure S1 of Supplementary information); information on molecular weight distribution was sought by GFC analysis. The molecular properties of the polymer are strongly related to both kefir culture conditions and kefiran extraction procedure. ${ }^{48-50} \mathrm{We}$ found $\mathrm{Mw}=4.32 \pm 0.44 \mathrm{MDa}$, in good agreement with Piermaria et al. $2008^{39}, \mathrm{R}_{\mathrm{g}}=88.5 \pm 8.9 \mathrm{~nm}$ and $\mathrm{A}_{2}=2.2$ 
( \pm 3.1$) 10^{-5} \mathrm{~cm}^{3} \mathrm{~mol} / \mathrm{g}^{2}$. Other kefiran variants, obtained by a purification process with a final trichloroacetic acid (TCA, 14\%) treatment, presented significantly lower molecular weights, lower values of $\mathrm{R}_{\mathrm{g}}$ and higher values of $\mathrm{A}_{2}$, indicating the presence of shorter and slightly anionic chains, resulting from mild oxidative degradation reactions. ${ }^{51}$ The higher values of $M_{w}$ and $R_{g}$ and the $A_{2}$ value close to zero are related to less oxidising extraction and purification conditions, yielding to longer and less hydrophilic polymer chains ${ }^{48,52}$

The gel filtration chromatogram, reported in Figure 1a, shows a single, almost Gaussian, molecular weight distribution. The values of the number average $(\mathrm{Mn})$ and weight average $(\mathrm{Mw})$ molecular weights, calculated using pullulan standards, are 2.17 MDa and 4.38 MDa, respectively (PDI=2). It is worth noting that the Mw values determined with the two techniques, SLS and GFC, are in excellent agreement confirming the suitability of pullulans as GFC standards.

A further confirmation of molecular structure and purity of the extracted polymer was sought by thermogravimetric (TGA) analysis. The thermogravimetric curve is reported in Figure 1b. The initial weight loss of polymer, between 55 and $150^{\circ} \mathrm{C}$, can be attributed to moisture (free and bound water representing ca. $10 \% \mathrm{w}$ ). The second inflection point of the thermogravimetric curve at $280{ }^{\circ} \mathrm{C}$, corresponding to a weight loss of about $3 \% \mathrm{w}$, may be due to the residual proteins in the system. The most prominent mass loss, with a main inflection point at $300{ }^{\circ} \mathrm{C}$, is due to the thermal degradation of the biopolymer. It is sharp and occurs at a temperature interval that is close to those reported in the literature. ${ }^{49,51}$ 

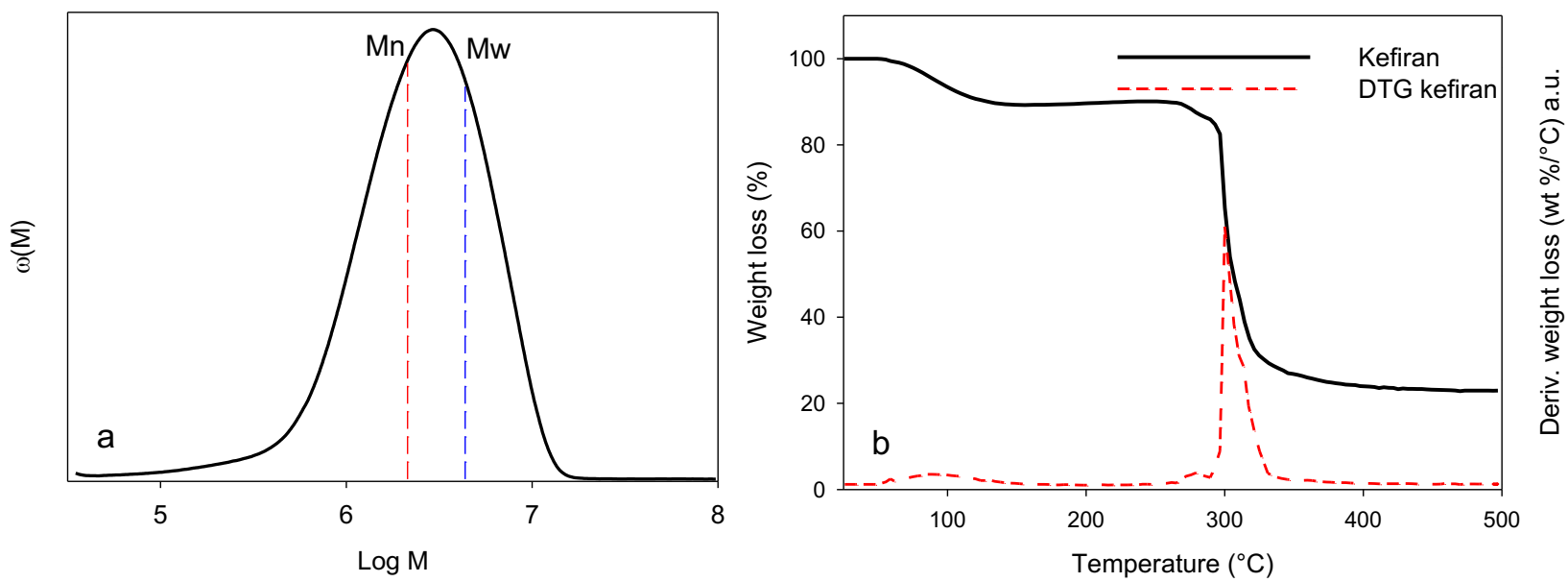

Figure 1. (a) Gel filtration chromatogram of $0.1 \%$ w kefiran aqueous solution, the number average and the weight average molecular weights are reported; (b) TGA analysis of freeze-dried kefiran.

\subsection{Shear viscosity and injectability of kefiran solutions}

In Figure 2a, the apparent viscosity as a function of shear rate is plotted for $\operatorname{Kef}(4), \operatorname{Kef}(5)$ and $\operatorname{Kef}(6)$ solutions. $\operatorname{Kef}(4)$ presents a Netwtonian plateau at low shear rates, followed by a decrease of viscosity at higher shear rates typical of the pseudoplastic behaviour, while $\operatorname{Kef}(5)$ and $\operatorname{Kef}(6)$ exhibit a pseudoplastic behavior in the whole shear rate range. For Kef(4), the entanglements that are disrupted by the imposed deformation can be reformed and no reduction in viscosity is observed at low shear rates. On the contrary, at higher rates of shear, when the rate of imposed deformation is higher than the rate of molecular rearrangements, chain entanglements loosen and the apparent viscosity decreases. At higher polymer concentration (e.g. for $\operatorname{Kef}(5)$ and $\operatorname{Kef}(6)$ ) the freedom of movement of the chain segments is more restricted and the pseudoplastic behavior arises already at low shear rates. Figure $2 \mathrm{~b}$ illustrates the mechanical spectra, obtained in small amplitude oscillation mode, of the three kefiran solutions. At low frequencies, all solutions exhibit the characteristic behaviour of viscoelastic liquids, with G" higher than G'. At higher frequencies, the time is not sufficient for the polymer chains to disentangle and G' curve approaches G" curve. Both moduli increase in value at the increase of polymer concentration. 

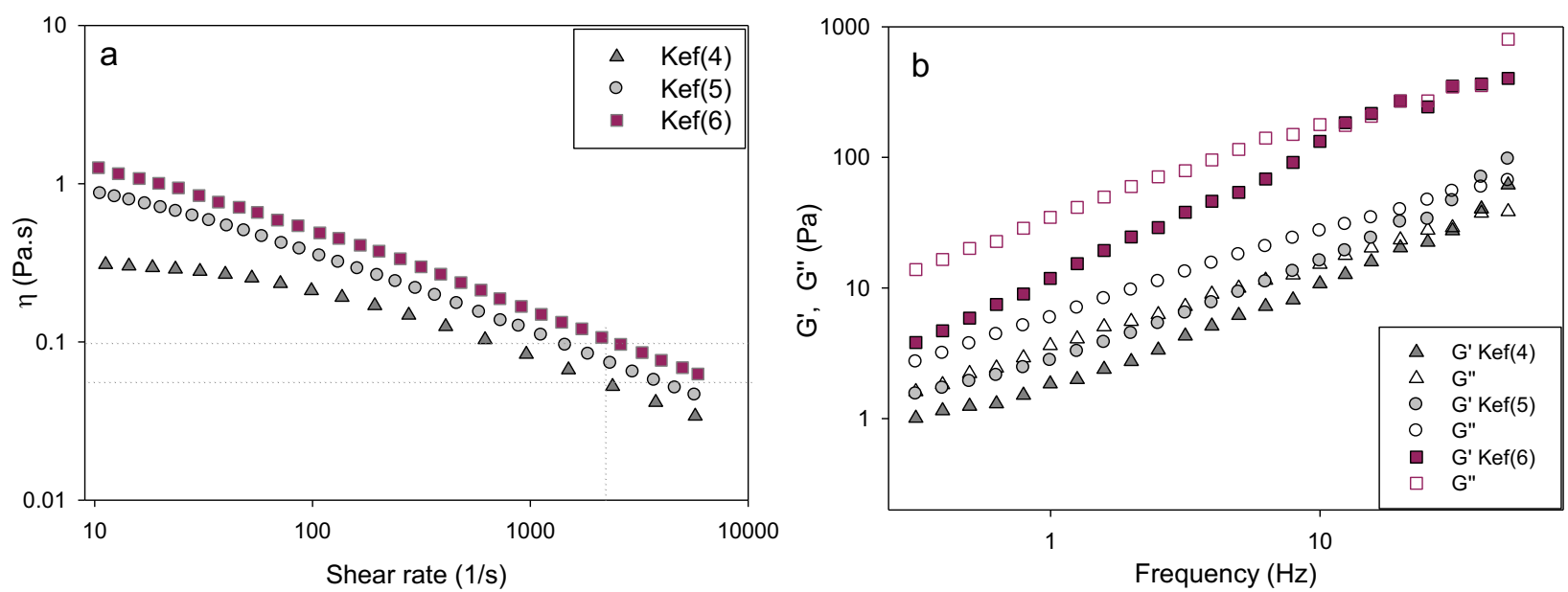

Figure 2. Viscoelastic properties of kefiran aqueous solutions at $25^{\circ} \mathrm{C}$. (a) Shear viscosity as a function of shear rate; (b) Storage (G') and loss (G’) shear moduli as a function of frequency.

The results reported in Table 2 show the injection time to extrude $1 \mathrm{ml}$ of solution at $25^{\circ} \mathrm{C}$ and the weight of the residual material in the syringe. The injection time varies from ca. 10 to $20 \mathrm{~s} / \mathrm{ml}$ that is within the range of commonly recommended injections rates.$^{53}$ From the dynamic viscosity value at $2000 \mathrm{~s}^{-1},{ }^{53}$ according to the viscosity-needle ID-glide force correlations shown by Watt et al. 2019,54 we would expect a glide force of about $10 \mathrm{~N},{ }^{54}$ that is lower than the highest recommended value of $20 \mathrm{~N}$ (ISO guidance 11608-3, sez. 4.3). The residual amount of material left over in the syringe after extrusion increases with concentration.

Table 2. Injection time to extrude $1 \mathrm{ml}$ of kefiran solutions and corresponding residual amount of material in the syringe after extrusion. Mean and standard deviations of ten trials per point.

\begin{tabular}{lll}
\hline System & Injection time $(\mathrm{s} / \mathrm{ml})$ & Residual amount $(\%)$ \\
\hline $\operatorname{Kef}(4)$ & $10.5 \pm 3$ & $3.2 \pm 0.3$ \\
$\operatorname{Kef}(5)$ & $14.5 \pm 3$ & $4.1 \pm 0.4$ \\
$\operatorname{Kef}(6)$ & $19.1 \pm 2$ & $4.8 \pm 0.6$ \\
\hline
\end{tabular}




\subsection{Gelation behaviour of kefiran aqueous solutions mixed with alcohols}

Aqueous solutions of kefiran form physical gels when they are mixed with ethanol or ethylene glycol and stored at low temperature for $24 \mathrm{~h} .{ }^{42}$ Other polysaccharides, such as xyloglucan ${ }^{55}$ and pectin ${ }^{56}$ that form physical gels when mixed with alcohols show a clear influence of the alcohol structure on the gelation process. For this reason, we performed a preliminary screening on four different alcohols, EtOH, EEE, Gro or PG, mixing them with $\operatorname{Kef}(4)$ solutions at 4:1 mass ratio (polymer solution to alcohol) and tilting the vials containing the mixtures at given time intervals to check if they were set into gels (see Figures S2a-d and S3a-b of Supporting information). We observed that the addition of monohydric alcohols, such as EtOH (Figure S2a) or EEE (Figure S2b), causes instantaneous formation of white, dense, gel lumps dispersed in a transparent liquid medium. After 30 minutes, these systems set into slightly hazy gels incorporating the initially formed white lumps. On the contrary, the addition of the dihydric alcohol, PG, to $\operatorname{Kef}(4)$ (Figure S2c), always in the same proportions, did not induce instant gelation. The system loses the ability to flow only after ca. $6 \mathrm{~h}$, when it sets into a slightly opalescent gel that does no longer change its physical appearance during the following $42 \mathrm{~h}$. Glycerol (Figure S2d) does not induce gelation in $48 \mathrm{~h}$ of observation. For Kef(5) and $\operatorname{Kef}(6)$ solutions the same trend was observed: monohydric alcohols were always yielding heterogeneous gels, while glycerol did not induce gelation. PG produced uniform gels in a time that was shorter the higher was the polymer concentration (Figure S3a-b). From this preliminary screening, PG was selected as gelling agent for its ability to form uniform and dimensionally stable kefiran gels that incorporate and retain all the water initially present in the system (no syneresis was observed).

The gelation of aqueous kefiran with PG was also investigated by rheological analysis. In particular, the time to gel and the gel strength were measured from oscillatory tests where the frequency was varied while the amplitude of the deformation was kept constant. For each system, these measurements were repeatedly carried out on the same sample to follow the time-dependence of 
storage and loss moduli and were also performed on different samples, molded into disks and incubated in unperturbed conditions for given time lengths, paying care that during storage the water loss was minimized.

In Figure $3 \mathrm{a}$, the time course of storage and loss moduli at $\mathrm{T}=25{ }^{\circ} \mathrm{C}$ and $1 \mathrm{~Hz}$ for $\mathrm{Kef}(4) / \mathrm{PG}$, $\operatorname{Kef}(5) / \mathrm{PG}$ and $\operatorname{Kef}(6) / \mathrm{PG}$ is reported. In this type of rheological measurements, the onset of gelation can be detected from the G'-G' curves crossover. $\operatorname{Kef}(4) / P G$ and $\operatorname{Kef}(5) / P G$ systems start with values of G" that are higher than those of G'. The G'-G" curves crossover occurrs in about $6 \mathrm{~h}$ for Kef(4)/PG and in ca. $4 \mathrm{~h}$ for $\operatorname{Kef}(5) / \mathrm{PG} . \operatorname{Kef}(5) / \mathrm{PG}$ reaches a plateau in ca. $24 \mathrm{~h}\left(\mathrm{G}_{\text {plateau }}^{\prime}=1750 \mathrm{~Pa}, 1 \mathrm{~Hz}\right)$. For $\operatorname{Kef}(4) / P G$, the measurements were interrupted after $16 \mathrm{~h}$ because this system was more susceptible to drying during the test than the others. For $\operatorname{Kef}(6) / \mathrm{PG}, \mathrm{G}$ ' is always higher than G', suggesting that gelation has already occurred either during mixing $(5 \mathrm{~min}$ ) or deposition on the rheometer plate (ca. $3 \mathrm{~min}$ ), or during the time elapsed for the acquisition of the first data point (10 $\mathrm{min})$. Moreover, in the investigated time range, G" curve is almost invariant with frequency while G' steadily increases to reach a plateau in ca. $20 \mathrm{~h}\left(\mathrm{G}_{\text {plateau }}=2000 \mathrm{~Pa}, 1 \mathrm{~Hz}\right)$. In good agreement with the tilting test, the onset of gelation is shorter the higher is the polymer concentration. ${ }^{57}$

In order to assess the impact of any eventual water evaporation during the tests and to compare the three systems after the same conditioning process, the mechanical spectra of samples that were produced into a mold and conditioned for $48 \mathrm{~h}$ at room temperature were collected (Figure $3 \mathrm{~b}$ ). For $\operatorname{Kef}(5) / P G$ and $\operatorname{Kef}(6) / P G, G^{\prime}$ is higher than G' and they are both invariant with frequency. G' is ca. $1500 \mathrm{~Pa}$ for $\operatorname{Kef}(5) / \mathrm{PG}$ and ca. $2200 \mathrm{~Pa}$ for $\operatorname{Kef}(6) / \mathrm{PG}$ : these values are close to the $\mathrm{G}_{\text {plateau }}$ values from the plots of Figure 3a. Kef(4)/PG shows significantly lower G' curve (ca. $230 \mathrm{~Pa}$ ) and G" values that are strongly dependent on frequency. Since $\mathrm{Kef}(4) / \mathrm{PG}$ requires long time to set into gel and forms a relatively weak gel, this system was rejected.

The development of gel strength with time at controlled temperature of $25^{\circ} \mathrm{C}$ and $37^{\circ} \mathrm{C}$ in unperturbed conditions was investigated for $\operatorname{Kef}(6) / P G$ (Figure 3c-d). At both temperatures, the G' curve progressively shifts towards higher values and becomes invariant with frequency; after 48 hours, G' 
becomes also invariant with the storage time $\left(G^{\prime}\right.$ and $G$ ” vs. frequency plots for each system are reported in Figure S4 of Supporting information). We notice that at $37^{\circ} \mathrm{C}$ (Figure $3 \mathrm{~d}$ ) the time for incipient gelation is higher (ca. $2 \mathrm{~h}$ for $\operatorname{Kef}(6) / \mathrm{PG}$ ) and the $\mathrm{G}$ ' values corresponding to full strength are lower (ca. $750 \mathrm{~Pa})$.

The observations made on the effect of the alcohol type, polymer concentration and temperature on the onset of gelation provide also hints about the mechanism of gelation of kefiran in hydro-alcoholic solutions. When added to the aqueous polymer solution, alcohol competes with water to form $\mathrm{H}$ bonds with the polymer. Each monohydric alcohol molecule that binds to the polymer chain causes a net loss of one hydroxyl group and the exposure of the hydrophobic portion of the alcohol to the surrounding water. The solvated polymer becomes more hydrophobic and this favours polymerpolymer association and formation of "condensed domains" with concurrent significant increase of local viscosity (jamming).$^{58,59} \mathrm{~A}$ di-hydric alcohol like propylene glycol has the possibility of establishing either one or two coordinated H-bonds (with two hydroxyl groups present on the same repeating unit or on different units of the same or of different chains). Only when forms two coordinated H-bonds, it reduces the chain hydrophilicity. Therefore, a di-hydric alcohol is relatively less effective than monohydric alcohols in triggering hydrophobic association and formation of condensed domains. Glycerol is too hydrophilic to promote hydrophobic association of kefiran chains. In conclusion, the addition of monohydric and dihydric alcohols drive the aqueous polymer solution into an instability region where the hindered mobility of the polymer chains is preventing phase separation. As proposed by Winter, gelation can be seen as a frustrated phase transition (jamming transition) and the rheological inverse of glass transition. ${ }^{60}$ "Condensed domains" formed by hydrophobic association are the crosslinking points of the formed physical network. In this framework, the observed increase in gel time and reduction of gel strength for gels forming at $37^{\circ} \mathrm{C}$ compared to those formed at $25^{\circ} \mathrm{C}$ can also be explained. In facts, at higher temperature hydrogen bonding interactions are weakened and the driving force for gelation is reduced. 

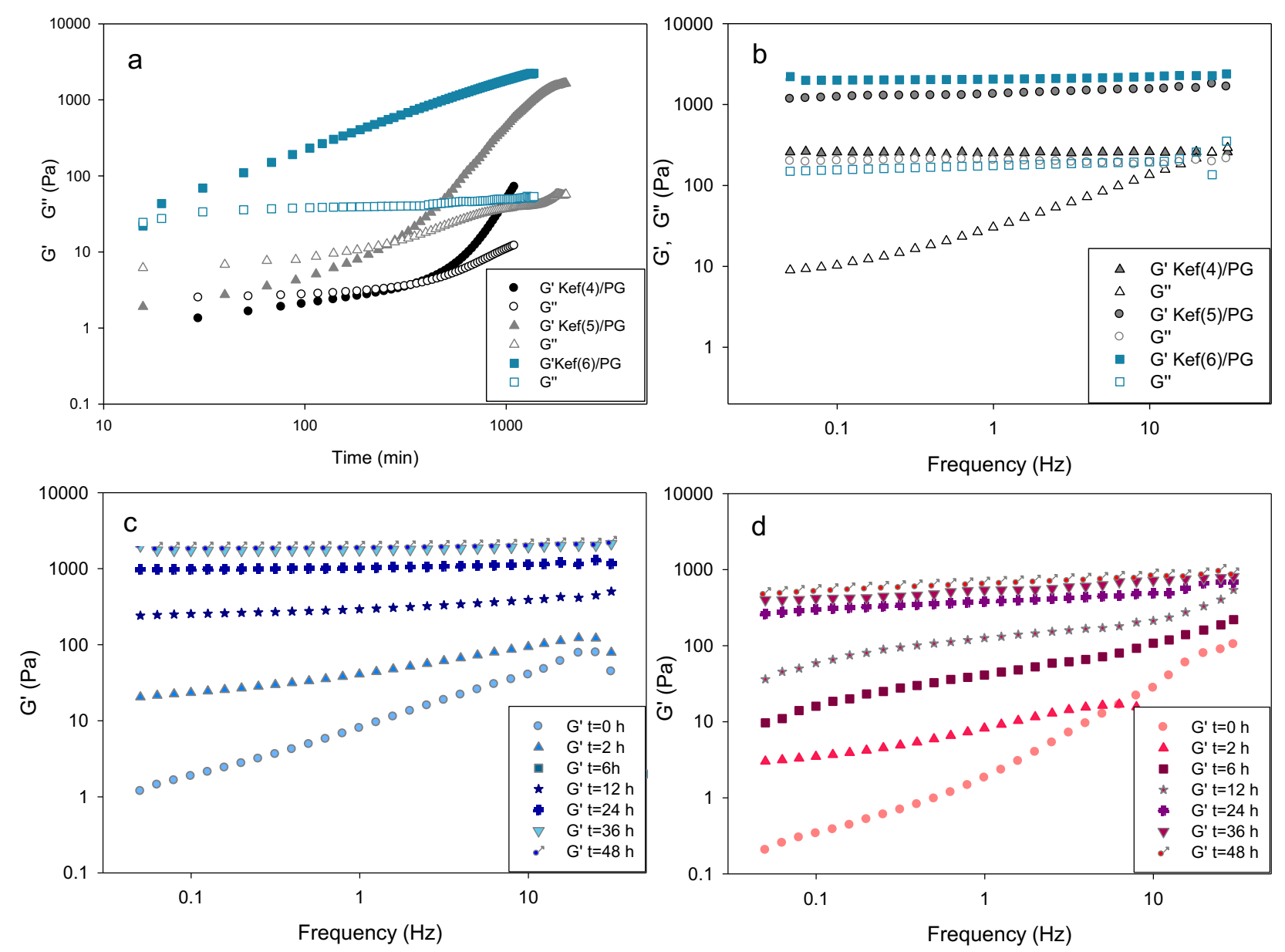

Figure 3. Viscoelastic properties of kefiran-propylene glycol gels. (a) G' and G" vs. time plots built extracting values at $1 \mathrm{~Hz}$ from consecutive frequency sweep runs carried out at $25^{\circ} \mathrm{C}$ for $\mathrm{Kef}(4) / \mathrm{PG}$, $\operatorname{Kef}(5) / P G$ and $\operatorname{Kef}(6) / P G ;$ (b) G' and G” vs. frequency plots for $\operatorname{Kef}(4) / P G, \operatorname{Kef}(5) / P G$ and Kef(6)/PG stored for $48 \mathrm{~h}$ at room temperature; G' and G', vs. frequency plots for Kef(6)/PG incubated for $1 \mathrm{~h}, 2 \mathrm{~h}, 6 \mathrm{~h}, 12 \mathrm{~h}, 24 \mathrm{~h}, 36 \mathrm{~h}$ and $48 \mathrm{~h}$ at $25^{\circ} \mathrm{C}(\mathrm{c})$, and $37^{\circ} \mathrm{C}(\mathrm{d})$.

A further practical conclusion of the rheological investigation is that it is advisable to store the gels at room temperature for 2 to 3 days before proceeding with any further characterization to ensure that the gelation process is completed.

\subsection{Hydrolytic degradation behavior of $\operatorname{Kef}(5) / P G$ and Kef(6)/PG gels}

The hydrolytic degradation of $\operatorname{Kef}(5) / \mathrm{PG}$ and $\operatorname{Kef}(6) / \mathrm{PG}$ has been studied by following the mass change of samples incubated at $37^{\circ} \mathrm{C}$ in isotonic PBS for up to 40 days and by investigating their 
eventual structural and morphological modification after the whole incubation period ("aged" samples). In Figure 4a, the relative mass change as a function of time is reported. Samples increase their mass during the first 5 days, then reach a plateau. After 20 days, their mass starts to slowly decrease. The maximum swelling degree is lower for $\operatorname{Kef}(6) / \mathrm{PG}$ (ca. 48\%) than for Kef(5)/PG (ca. 57\%), confirming that a higher polymer concentration leads to a tighter network, as also suggested by the mechanical spectra of the two systems. The mass decrease can be attributed to erosion or molecular degradation of the polymer and/or to the rearrangement of the polymer chains into a tighter network.

FTIR analysis and SEM investigation of the freeze-dried systems were carried out to identify the most probable cause of the observed mass decrease. For these analyses, samples were carefully rinsed with water to remove unbound alcohol and salts, and freeze-dried. In figure $4 \mathrm{~b}$, the FTIR spectra of $\operatorname{Kef}(5) / P G$ and "aged $\operatorname{Kef}(5) / P G$, together with the spectrum of the extracted kefiran polymer as reference, are reported. The polymer shows an intense broad peak around $3430 \mathrm{~cm}^{-1}$ characteristic of the stretching vibration of hydroxyl groups; a weak band at $2935 \mathrm{~cm}^{-1}$ due to methylene $\mathrm{C}-\mathrm{H}$ stretching; a peak at $1650 \mathrm{~cm}^{-1}$ with a shoulder at $1730 \mathrm{~cm}^{-1}$ associated to $\mathrm{C}=\mathrm{O}$ stretching vibrations of aldehyde and carboxyl groups; a broad stretching band in the $1000-1200 \mathrm{~cm}^{-1}$ region for $\mathrm{C}-\mathrm{O}-\mathrm{C}$ and $\mathrm{C}-\mathrm{O}$ ethers; and peaks at 878 and $900 \mathrm{~cm}^{-1}$ corresponding to the $\beta$-D-glucopyranoside and $\alpha$ D-galactopyranosyl rings, respectively. $\operatorname{Kef}(5) / \mathrm{PG}$ gel shows the same peaks as kefiran. Both hydroxyl groups and ethers vibration bands are attenuated, probably due to their involvement in hydrogen bonds. Additional small peaks at $2970 \mathrm{~cm}^{-1}, 927 \mathrm{~cm}^{-1}, 838 \mathrm{~cm}^{-1}$ are attributable to bound PG. Aging of the gel in PBS at $37^{\circ} \mathrm{C}$ for 40 days causes the disappearance of the characteristic PG peaks and further attenuation of the ethers band. $\operatorname{Kef}(6) / \mathrm{PG}$ samples before and after aging (spectra not reported for brevity) do not show appreciable differences with the corresponding spectra of $\operatorname{Kef}(5) / P G$. In the light of these results we can conclude that no significant chemical modification of kefiran is occurring during incubation. 

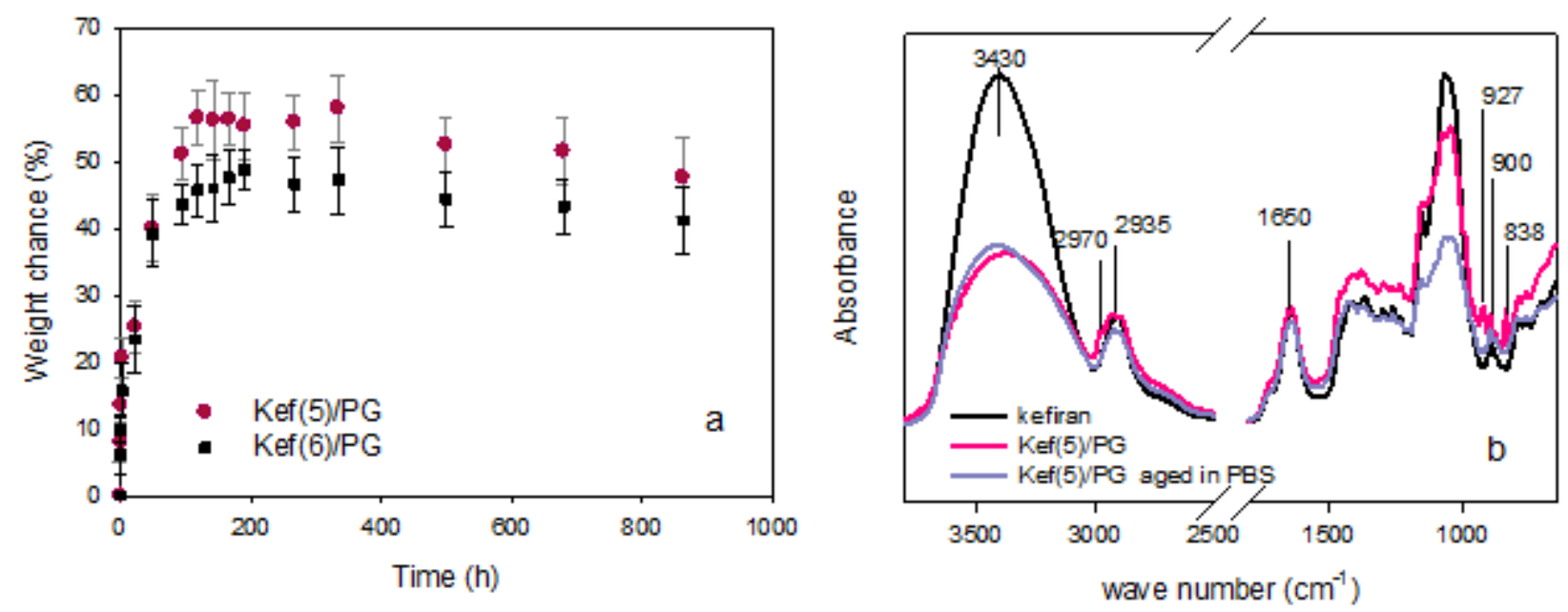

Figure 4. (a) Mass change as function time for $\operatorname{Kef}(5) / \mathrm{PG}$ and $\operatorname{Kef}(6) / \mathrm{PG}$. (b) FTIR spectra of Kef(5)/PG and "aged" Kef(5)/PG gels.

The SEM morphology of the freeze-dried samples $\operatorname{Kef}(5) / \mathrm{PG}$ and $\operatorname{Kef}(6) / \mathrm{PG}$, before and after aging, is shown in Figure 6a-f. When analyzed after preparation and conditioning at room temperature for $48 \mathrm{~h}$, both systems are characterized by a uniform distribution of relatively wide pores (10-30 $\mu \mathrm{m})$. Kef(6)/PG (Figure 6b and 6d) generally presents slightly smaller pores with thicker walls. 

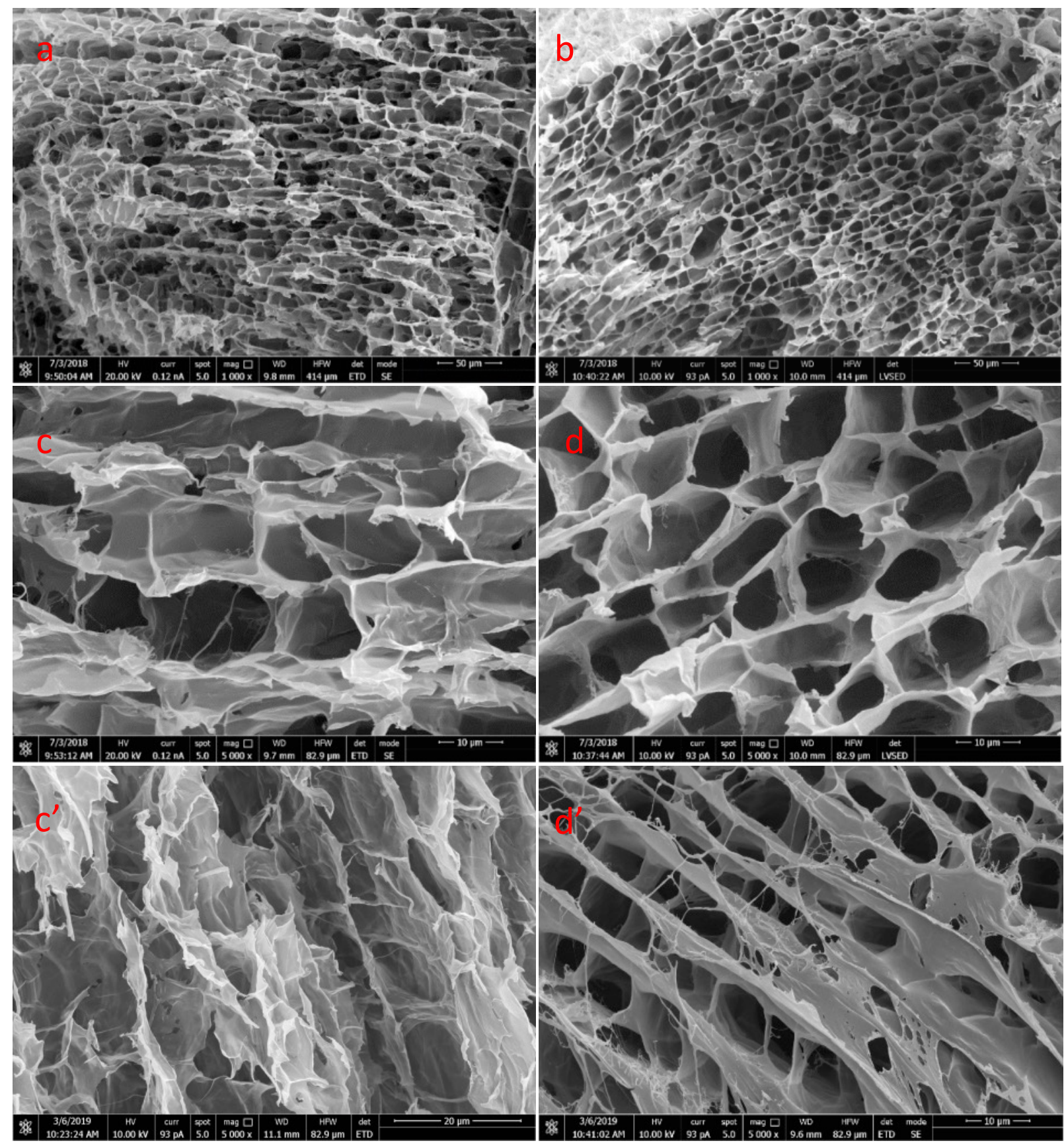

Figure 6. Scanning electron micrographs of (a, c) $\operatorname{Kef}(5) / \mathrm{PG}$ and (b, d) $\operatorname{Kef}(6) / \mathrm{PG}$ at two different magnifications; (c') "aged” Kef(5)/PG and (d') “aged” Kef(6)/PG.

After 40 days aging, the porosity is not dramatically altered in size (see Figures $6 c^{\prime}$-d'). Yet, a tendency of the systems to develop a more compact, layered structure with stacked sheets connected by filaments and transverse walls is noted, especially for $\operatorname{Kef}(6) / \mathrm{PG}$. For $\operatorname{Kef}(5) / \mathrm{PG}$, the layered structure is probably partially collapsed upon freeze-drying. We can argue that the prolonged 
incubation in water and extraction of the residual alcohol molecules induces a further spontaneous rearrangement of the polymer chains. A similar tendency to develop a layered morphology upon incubation in water has been observed for gels of partially degalactosilated xyloglucan ${ }^{61}$ and enzymatically-degraded xylans ${ }^{62}$.

\subsection{Incorporation and release of model molecules}

In order to test the ability of these systems to release or retain guest molecules of very different size, and as a way to probe the network mesh size and pores interconnectivity, $\mathrm{Kef}(5) / \mathrm{PG}$ and $\mathrm{Kef}(6) / \mathrm{PG}$ were prepared with the addition of two model molecules, a hydrophilic fluorescent probe (Atto 633) and FITC-labeled insulin. Both the loaded gels were immersed in a small volume of PBS buffer, collected and replaced with fresh solvent at regular intervals, and the desorption of the loaded molecule from the gel was followed as a function of the time for $24 \mathrm{~h}$. As shown in Figure 7, the incorporated molecular probe is only partially released from both systems (ca. $60 \%$ and ca. $50 \%$ of the loaded amount for $\operatorname{Kef}(5) / \mathrm{PG}$ and $\operatorname{Kef}(6) / \mathrm{PG}$, respectively) and always in about $3 \mathrm{~h}$, while the remaining loaded amount is irreversibly entrapped in the gel. The release of Atto 633 in terms of

mass fraction of drug released with time follows a power-law relationship, $\frac{M_{t}}{M_{\infty}}=t^{n}$, with $n=0.5$, therefore consistent with a Fickian transport mechanism, that supports the presence of interconnected porosity. ${ }^{63}$

On the contrary, no evidence of released insulin is provided by both absorption and emission spectroscopies carried out on samples of the receiving phases collected from both systems over $24 \mathrm{~h}$. The formation of insulin aggregates on hydrophobic material surfaces is a well-known phenomenon. ${ }^{64}$ Therefore, we expect insulin to aggregate and adsorb on the hydrophobic domains of the gel. Longterm release studies with therapeutically-relevant molecules and conditions will be carried out in the immediate future. 


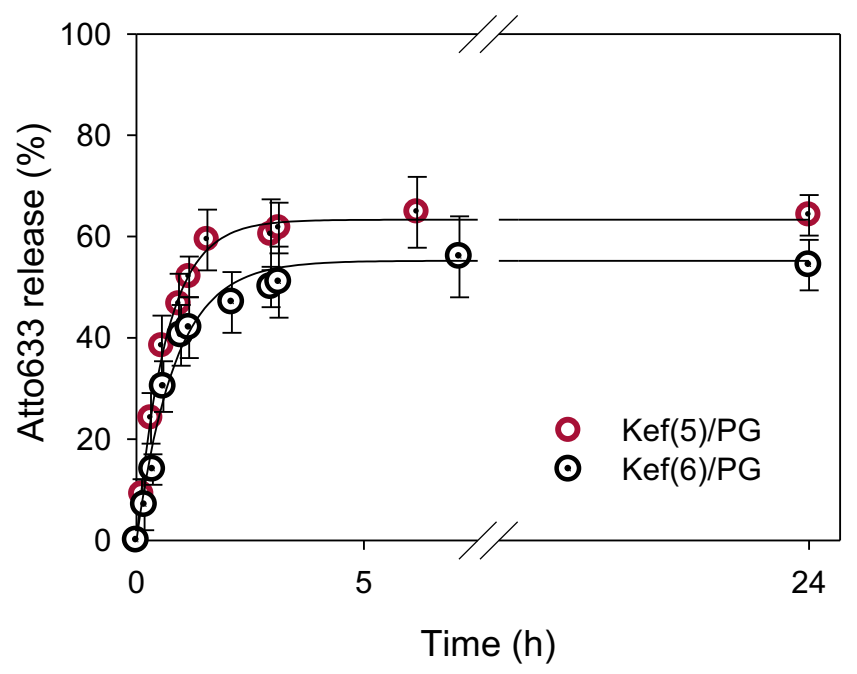

Figure 7. Cumulative Atto $633(\%)$ released from $\operatorname{Kef}(5) / \mathrm{PG}$ and $\operatorname{Kef}(6) / \mathrm{PG}$ gels.

\subsection{Cell viability}

A preliminary investigation of kefiran gels biocompatibility was performed via in vitro cell viability and cytotoxicity tests. For these evaluations the gel produced with the higher amount of kefiran (Kef(6)/PG) was used, considering the exposure of cells to the most concentrated system as the most challenging condition. Figure 8 shows the results of the Alamar Blue assay carried out with preosteoblastic MC3T3-E1 cells incubated for 1, 2, 4, 8 and 12 days in the presence of Kef(6)/PG (Fig. 8a) and the fluorescence microscopy cells imaging after 2, 4 and 8 days of incubation (Figure 8b-d). It is evident that kefiran gel does not affect cell proliferation: the growth curve of cells that are in direct contact to the gel or are exposed to leachable chemicals that present does not differ from the control system one and the morphology of those cells is also unaltered. Fluorescence microscopy analysis (see Figure 9a-d) after the acridine orange staining, that is a specific die for the detection of apoptotic damage, also supports the absence of cytotoxic effects induced by the presence of the in the culture medium. These preliminary results encourage to continue the biological evaluation of these systems as scaffolds for tissue regeneration or implantable drug delivery devices. 

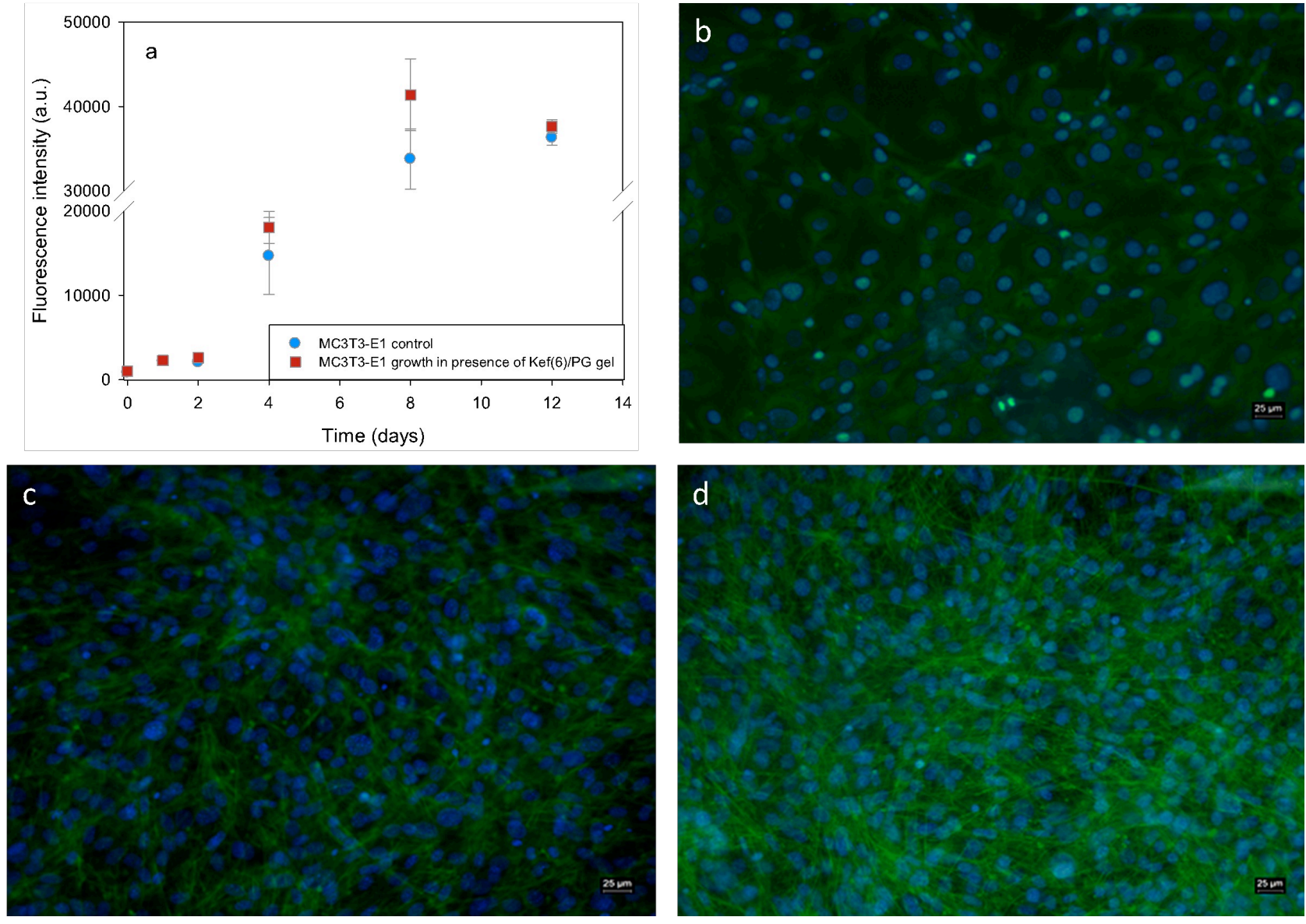

Figure 8. (a) Proliferation assay of MC3T3-E1 cells growth in 2D condition in culture medium for the control and in culture medium in presence of $\operatorname{Kef}(6) / \mathrm{PG}$ gel. (b) MC3T3-E1 cells growth in 2D condition in presence of $\operatorname{Kef}(6) / P G$ (b) for 2 days, (c) for 4 days, (d) for 8 days. Cytoskeleton was stained with Phalloidin ${ }^{\mathrm{FITC}}$ (green); nucleus was stained with DAPI (blue). Magnification was 20x. Bar is $25 \mu \mathrm{m}$. 

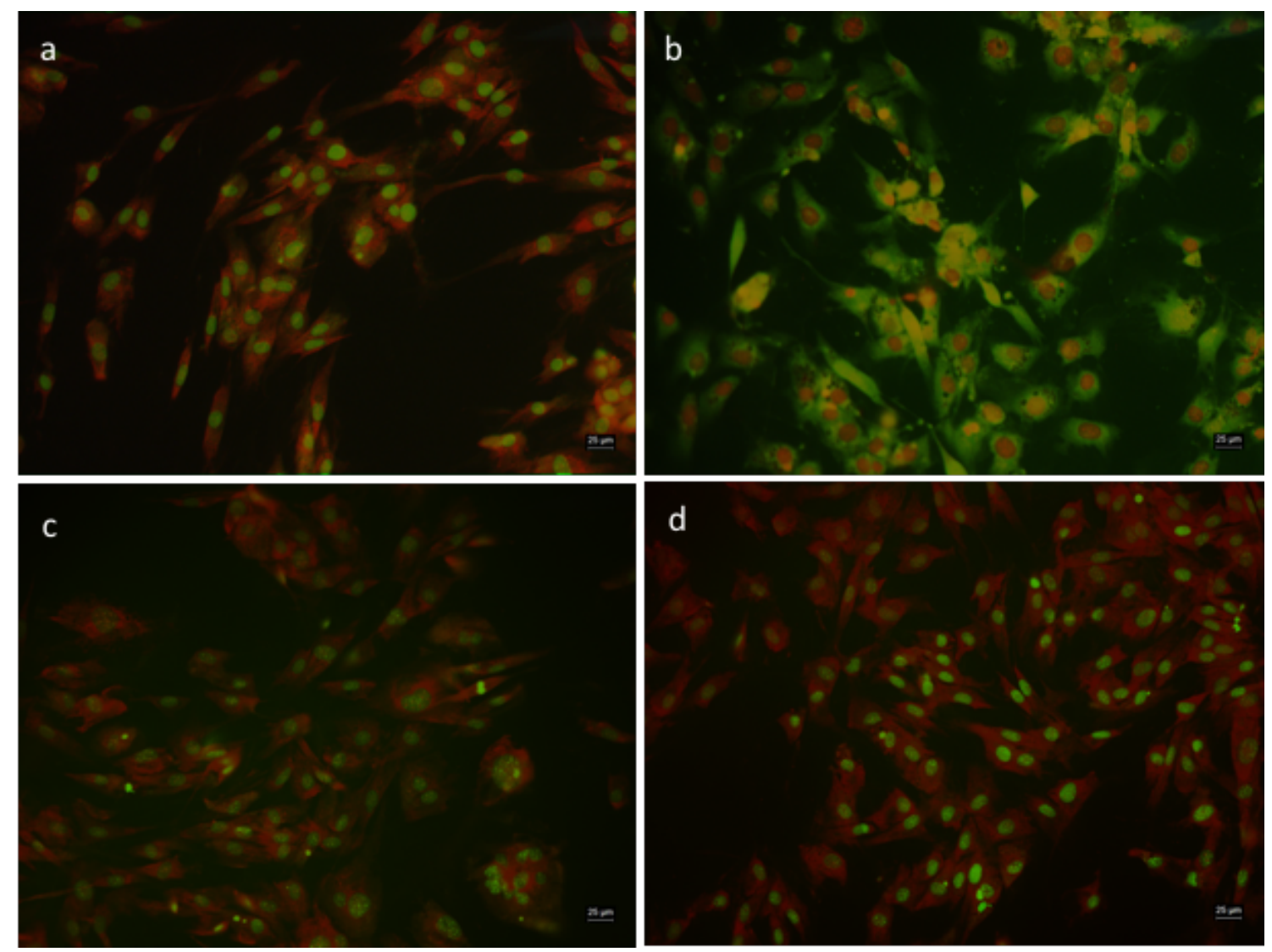

Figure 9. Acridine Orange staining (a) Negative control; (b) Positive control; Cells grown in presence of $\operatorname{Kef}(6) / P G$ gel (c) for 4 days (d) for 8 days. Magnification was 20x. Bar is $25 \mu \mathrm{m}$.

\section{CONCLUSIONS}

Kefiran aqueous solutions in the concentrated regime (4-6\%w) can be injected through small gauge (G26) syringe needles with acceptable glide forces and leaving low amount $(<5 \%$ w) of residual material in the syringe. When they are mixed with PG, at 4:1 mass ratio, these solutions yield homogeneous gels in a time that is compatible with patient immobilization (the shorter the higher is the polymer concentration) and do not undergo syneresis. When incubated in isotonic buffer solutions at $37^{\circ} \mathrm{C}$, these gels swell increasing their mass up to a maximum of $60 \%$ their initial weight during the first 5 days, then they start to slowly loose mass, most probably water. While the polymer is not appreciably chemically modified upon prolonged incubation, the residual alcohol is leached out of the gel and the morphology evolves from a random interconnected pores structure to a layered structure. The observations made using various alcohols, together with the dynamic rheological 
characterization of kefiran solutions mixed with PG, varying polymer concentration and temperature, provided interesting hints for the proposition of a possible mechanism of gelation that will be corroborated by further fundamental studies.

Incorporation of larger guest molecules in these gels may lead to their efficient entrapment, as observed for insulin, while smaller and more hydrophilic molecules can be partially released by diffusion through the inner aqueous phase filling the pores of the structure, as observed for Atto 633. Both the possibility of enabling the diffusion of smaller molecules (e.g. nutrients) and the entrapment of larger biomolecules (e.g. growth factors) are desirable features of instructive scaffolds for tissue engineering interventions. The absence of toxic effects on cells placed in direct contact with the gel encourage to pursue the biological evaluation of kefiran/propylene glycol-water formulations as injectable, in-situ forming scaffolds or implantable delivery devices.

\section{REFERENCES}

[1] Huebsch, N.; Mooney, D.J. Inspiration and application in the evolution of biomaterials. Nature 2009, 462, 426-432.

[2] Balakrishnan, B.; Banerjee, R. Biopolymer-Based Hydrogels for Cartilage Tissue Engineering. Chem. Rev. 2011, 111, 4453-4474.

[3] Helary, C.; Desimone, M.F. Recent advances in biomaterials for tissue engineering and controlled drug delivery. Curr. Pharm. Biotechnol. 2015, 16, 635-645.

[4] Davis, H.E.; Leach, J.K. Designing Bioactive Delivery Systems for Tissue Regeneration Ann. Biomed. Eng. 2011, 39(1), 1-13.

[5] Ricca, M.; Foderà, V .; Giacomazza, D.; Leone, M.; Spadaro, G.; Dispenza, C. Probing the internal environment of PVP networks generated by irradiation with different sources. Colloid Polym. Sci.2010, 288, 969-980.

[6] Dispenza, C.; Todaro, S.; Bulone, D.; Sabatino, M.A.; Ghersi, G.; San Biagio, P.L.; Lo Presti, C. Physico-chemical and mechanical characterization of in-situ forming xyloglucan gels 
incorporating a growth factor to promote cartilage recontruction. Mater. Sci. Eng. C 2017, 70, $745-752$.

[7] Slaughter, B.V.; Khurshid, S.S.; Fisher, O.Z.; Khademhosseini, A.; Peppas, N.A. Hydrogels in regenerative medicine. Adv. Mater. 2009, 21, 3307-3329.

[8] Polo-Corrales, L.; Latorre, Esteves M.; Ramirez-Vick, JE. Scaffold design for bone regeneration. J. Nanosci. Nanotechnol. 2014, 14, 15-56.

[9] Burdick, J.A.; Prestwich, G.D. Hyaluronic acid hydrogels for biomedical applications. Adv. Mater. 2011, 23, H41-56.

[10] Balakrishnan, B.; Joshi, N.; Jayakrishnan, A.; Banerjee, R. Self-crosslinked oxidized alginate/gelatin hydrogel as injectable, adhesive biomimetic scaffolds for cartilage regeneration. Acta Biomater. 2014, 10(8), 3650-3663.

[11] Navaei, A.; Truong, D.; Heffernan, J.; Cutts, J.; Brafman, D.; Sirianni, R.W.; Vernon, B.; Nikkhah, M. PNIPAAm-based biohybrid injectable hydrogel for cardiac tissue engineering. Acta Biomater. 2016, 32, 10-23.

[12] Yan, C.; Altunbas, A.; Yucel, T.; Nagarkar, R.P.; Schneider, J.P.; Pochan D.J. Injectable solid hydrogel: mechanism of shear-thinning and immediate recovery of injectable $\beta$-hairpin peptide hydrogels. Soft Matter 2010, 6(20), 5143-5156.

[13] Li, Y.; Rodrigues, J.; Toma', H. Injectable and biodegradable hydrogels: gelation, biodegradation and biomedical applications. Chem. Soc. Rev. 2012, 41, 2193-2221.

[14] Kempe, S.; Mäder K. In situ forming implants - an attractive formulation principle for parenteral depot formulations J. Controll. Release 2012, 161(2), 668-679.

[15] Klouda, L.; Mikos, A.G. Thermoresponsive hydrogels in biomedical applications - a review. Eur. J. Pharm. Biopharm. 2008, 68, 34-45.

[16] Todaro, S.; Sabatino, M.A.; Walo, M.; Mangione, M.R.; Bulone, D.; Dispenza, C. Influence of gamma-irradiation on thermally induced mesoscopic gelation of degalactosylated xyloglucan. Radiat. Phys. Chem. 2014, 94, 245-248. 
[17] Todaro, S.; Sabatino, M.A.; Mangione, M.R.; Picone, P.; Di Giacinto, M.L.; Bulone, D.; Dispenza, C. Temporal control of xyloglucan self-assembly into layered structures by radiationinduced degradation. Carbohydr. Polym. 2016, 152, 382-390.

[18] Liu, L.; Gao, Q.; Lu, X.; Zhou, H. In situ forming hydrogels based on chitosan for drug delivery and tissue regeneration. Asian J. Pharm. Sci. 2016, 11, 673-683.

[19] Hannouche, D.; Terai, H.; Fuchs, J.R.; Terada, S.; Zand, S.; Nasseri, B.A.; Petite, H.; Sedel, L.; Vacanti, J.P. Engineering of implantable cartilaginous structures from bone marrow-derived mesenchymal stem cells. Tissue Eng. 2007, 13, 87.

[20] Montembault, A.; Tahiri, K.; Korwin-Zmijowska, C.; Chevalier, X.; Corvol, M.T.; Domard, A. A material decoy of biological media based on chitosan physical hydrogels: application to cartilage tissue engineering. Biochimie 2006, 88,551 .

[21] Giacomazza, D.; Sabatino, M.A.; Catena, A.; Leone, M.; San Biagio, P.L.; Dispenza, C. Maltose conjugated chitosans induce macroscopic gelation of pectin solutions at neutral $\mathrm{pH}$. Carbohydr. Polym. 2014, 114, 141-148.

[22] Todaro, S.; Sabatino, M.A.; Ajovalasit, A.; Ditta, L.A.; Castiglia, D.; Wach, R.A.; Ulanski, P.; Bulone, D.; Dispenza, C. Radiation engineering of xyloglucan hydrogels. Chem. Eng. Trans. 2016, 49, 289-294.

[23] Yang, J.A.; Yeom, J.; Hwang, B.W.; Hoffman, A.S.; Hahn, S.K. In situ-forming injectable hydrogels for regenerative medicine. Prog. Polym. Sci. 2014, 39, 1973-1986.

[24] Nisbet, D.R.; Rodda, A.E.; Horne, M.K.; Forsythe, J.S.; Finkelstein, D.I. Implantation of Functionalized Thermally Gelling Xyloglucan Hydrogel Within the Brain: Associated Neurite Infiltration and Inflammatory Response. Tissue Eng. Part A 2010, 16(9) http://doi.org/10.1089/ten.tea.2009.0677.

[25] Yan, S.; Wang, T.; Feng, L.; Zhu, J.; Zhang, K.; Chen, X.; Cui, L.; Yin, J. Injectable in situ selfcross-linking hydrogels based on poly(L-glutamic acid) and alginate for cartilage tissue engineering. Biomacromolecules 2014, 15, 4495-4508. 
[26] Naahidi, S.; Jafari, M.; Logan, M.; Wang, Y.; Yuan, Y.; Bae, H.; Dixon, B.; Chen, P. Biocompatibility of hydrogel-based scaffolds for tissue engineering applications. Biotechnol $A d v .2017,35,530-544$.

[27] Micheli, L.; Uccelletti, D.; Palleschi, C.; Crescenzi, V. Isolation and characterisation of a ropy Lactobacillus strain producing the exopolysaccharide kefiran.Appl.Microbiol.Biotechnol.1999, $53,69-74$.

[28] Ghasemlou, M.; Khodaiyan, F.; Jahanbin, K.; Gharibzahedi, S.M.T.; Taheri S. Structural investigation and response surface optimisation for improvement of kefiran production yield from a low-cost culture medium. Food Chem. 2012, 133, 383-389.

[29] Exarhopoulos, S.; Raphaelides, S.N.; Kontominas, M.G. Conformational studies and molecular characterization of the polysaccharide kefiran. Food Hydrocolloids 2018, 77, 347e356

[30] Toscano, M.; Carfi Pavia, F.; Conoscenti, G.; Sabatino, M.A.; La Carrubba, V.; Dispenza, C.; Brucato, V. Kefiran-based scaffolds for biomedical applications. Chem. Eng. Trans. 2018, 64, $181-186$

[31] Huang, Y.; Wang, X.; Wang, J.; Wu, F.; Sui, Y.; Yang, L.; Wang, Z. Lactobacillus plantarum strains as potential probiotic cultures with cholesterol-lowering activity. J. Dairy Sci. 2013, 96, $2746-2753$

[32] Lee, M.Y.; Ahn, K.S.; Kwon, O.K.; Kim, M.J.; Kim, M.K.; Lee, I.Y.; Oh, S.R.; Lee, H.K. Antiinflammatory and anti-allergic effects of kefir in a mouse asthma model. Immunobiology 2007, $212,647-654$

[33] Rodrigues, K.L.; Caputo, L.R.G.; Carvalho, J.C.T.; Evangelista, J.; Schneedorf, J.M. Antimicrobial and healing activity of kefir and kefiran extract. Int. J. Antim. Agents 2005, 25, 404-408.

[34] Ghasemlou, M.; Khodaiyan, F.; Oromiehie A.; Physical, mechanical, barrier, and thermal properties of polyol-plasticized biodegradable edible film made from kefiran. Carbohydr. Polym. 2011, 84, 477-483. 
[35] Rizk, S.; Maalouf, K.; Baydoun, E. The antiproliferative effect of kefir cell-free fraction on Hut102 malignant T lymphocytes. Clin. Lymphoma Myeloma 2009, 9, 198-203.

[36] LeBlanc, A.; Matar, C.; Farnworth, E.; Perdigon, G. Study of cytokines involved in the prevention of a murine experimental breast cancer by kefir. Cytokine 2006, 34, 1-8.

[37] Radhouani, H.; Da Mota Martins Goncalves, C.; Antunes De Oliveira J.M. Kefiran for use in regenerative medicine and/or tissue engineering. 2018 PATENT WO2018/042405 A1.

[38] Radhouani, H.; Gonçalves, C.; Maia, F.R.; Oliveira, J.M.; Reis, R.L. Biological performance of a promising Kefiran-biopolymer with potential in regenerative medicine applications: a comparative study with hyaluronic acid. J.Mater. Sci.: Mater.Med. 2018, 29(8),124.

[39] Piermaria, J.A.; de la Canal, M.L.; Abraham, A.G. Gelling properties of kefiran, a food-grade polysaccharide obtained from kefir grain. Food Hydrocolloids 2008, 22, 1520-1527.

[40] Zavala, L.; Roberti, P.; Piermaria, J.A.; Abraham, A.G. Gelling ability of kefiran in the presence of sucrose and fructose and physicochemical characterization of the resulting cryogels. J. Food Sci Technol. 2015, 52(8), 5039-5047.

[41] Kazazi, H.; Khodaiyan, F.; Rezaei, K.; Pishvaei, M.; Mohammadifar, M.A.; Moieni, S. Rheology and microstructure of kefiran and whey protein mixed gels. J. Food Sci Technol. 2017, 54(5) 1168-1174.

[42] Mukai, T.; Watanabe, N.; Toba, T.; Itoh, T.; Adachi, S. Gel-forming characteristics and rheological properties of kefiran. J. Food Sci., 1991, 56, 1017-1026.

[43] Strickley R.G. Solubilizing excipient in oral and injectable formulations Pharm. Res. 2004, 21(2), 201-229.

[44] Piermaria, J.A.; Pinotti, A.; Garcia, M.A.; Abraham, A.G. Films based on kefiran, an exopolysaccharide obtained from kefir grain: development and characterization. Food Hydrocolloids 2009, 23, 684-90.

[45] Starcher, B. A ninhydrin-based assay to quantitate the total protein content of tissue samples, Anal Biochem. 2001, 292, 125-129. 
[46] Stepanek, P. Data Analysis in Dynamic Light Scattering. In Dynamic Light Scattering: The Method and Some Applications; Brown, W., Ed.; Oxford University Press: Oxford, U.K., 1993; pp 177-240.

[47] ASTM Standard F2900:2011, Standard Guide for Characterization of Hydrogels used in regenerative Medicine, ASTM International, West Conshohocken, PA, USA, 2011.

[48] Yang, L.; Zhang, L.M. Chemical structural and chain conformational characterization of some bioactive polysaccharides isolated from natural sources. Carbohydr. Polym. 2009, 76, 349-361.

[49] Zajšek, K.; Goršek, A.; Kolar, M. Cultivating Conditions Effects on Kefiran Production by the Mixed Culture of Lactic Acid Bacteria Imbedded within Kefir Grains. Food Chem. 2013, 139(14), 970-977.

[50] Pop, C.R.; Salanţă, L.; Rotar, A.M.; Semeniuc, C.A.; Socaciu, C.; Sindic M. Influence of extraction conditions on characteristics of microbial polysaccharide kefiran isolated from kefir grains biomass J. Food Nutr. Res. 2016, 55(2), 121-130

[51] Ahmed, Z.; Wang, Y.; Anjum, N.; Ahmad, A.; Khan S.T. Characterization of exopolysaccharide produced by Lactobacillus kefiranofaciens ZW3 isolated from Tibet kefir - Part II. Food Hydrocolloids 2013, 30, 343-350.

[52] Xu, X.; Chen, P.V.; Wang, Y.; Zhang, L. Chain conformation and rheological behavior of an extracellular heteropolysaccharide Erwinia gum in aqueous solution. Carbohydr. Res. 2009, 344, $113-119$.

[53] Allmendinger, A.; Fischer, S.; Huwyler, J.; Mahler, H.-C.; Schwarb, E.; Zarraga, I.E.; Mueller, R. Rheological characterization and injection forces of concentrated protein formulations: An alternative predictive model for non-Newtonian solutions. Eur. J. Pharm. Biopharm. 2014, 87, $318-328$.

[54] Watt, R.P.; Khatri, H.; Dibble, A.R.G. Injectability as a function of viscosity and dosing materials for subcutaneous administration. Int.J. Pharm. 2019, 554, 376-386. 
[55] Yuguchi, Y.; Kumagai, T.; Wu, M.; Hirotsu, T.; Hosokawa J. Gelation of xyloglucan in water/alcohol systems. Cellulose 2004, 11, 203-208.

[56] Oakenfull, D.; Scott, A. Hydrophobic interaction in the gelation of high methoxyl pectins. $J$. Food Sci. 1984, 49 (4), 1093-1098.

[57] Ross-Murphy, B. Gelation Kinetics - Problems and Prospects. J. Macromol. Sci. B 2005, 44(6), 1007-1019.

[58] Trappe, V.; Prasad, V.; Cipalletti, L.; Segre, P.N.; Weitz, D.A. Jamming phase diagram for attractive particles. Nature 2001, 411, 772-775.

[59] Hagan, M.F.; Elrad, O.M.; Jack, R.L. Mechanisms of kinetic trapping in self-assembly and phase transformation. J. Chem. Phys. 2011, 135, 104115.

[60] Winter, H.H. Glass transition as the rheological inverse of gelation. Macromolecules 2013, 46 (6), 2425-2432.

[61] Todaro, S.; Sabatino, M.A.; Mangione, M.R.; Picone, P.; Di Giacinto, M.L.; Bulone, D.; Dispenza, C. Temporal control of xyloglucan self-assembly into layered structures by radiationinduced degradation, Carbohydr. Polym. 2016, 152, 382-390.

[62] Köhnke, T.; Lin, A.; Elder, T.; Theliander, H.; Ragauskas A.J. Nanoreinforced xylan-cellulose composite by freeze-casting. Green Chem. 2012, 14, 1864-1869.

[63] Li, J.; Mooney, D.J. Designing hydrogels for controlled drug delivery. Nat. Rev. Mater. 2016, 1(12), 16071, DOI: 10.1038/natrevmats.2016.71.

[64] Nault, L.; Guo P.; Jain, B.; Bréchet, Y.; Bruckert, F.; Weidenhaupt, M. Human insulin adsorption kinetics, conformational changes and amyloidal aggregate formation on hydrophobic surfaces. Acta Biomater. 2013, 9(2), 5070-5079.

\section{Supplementary Information}




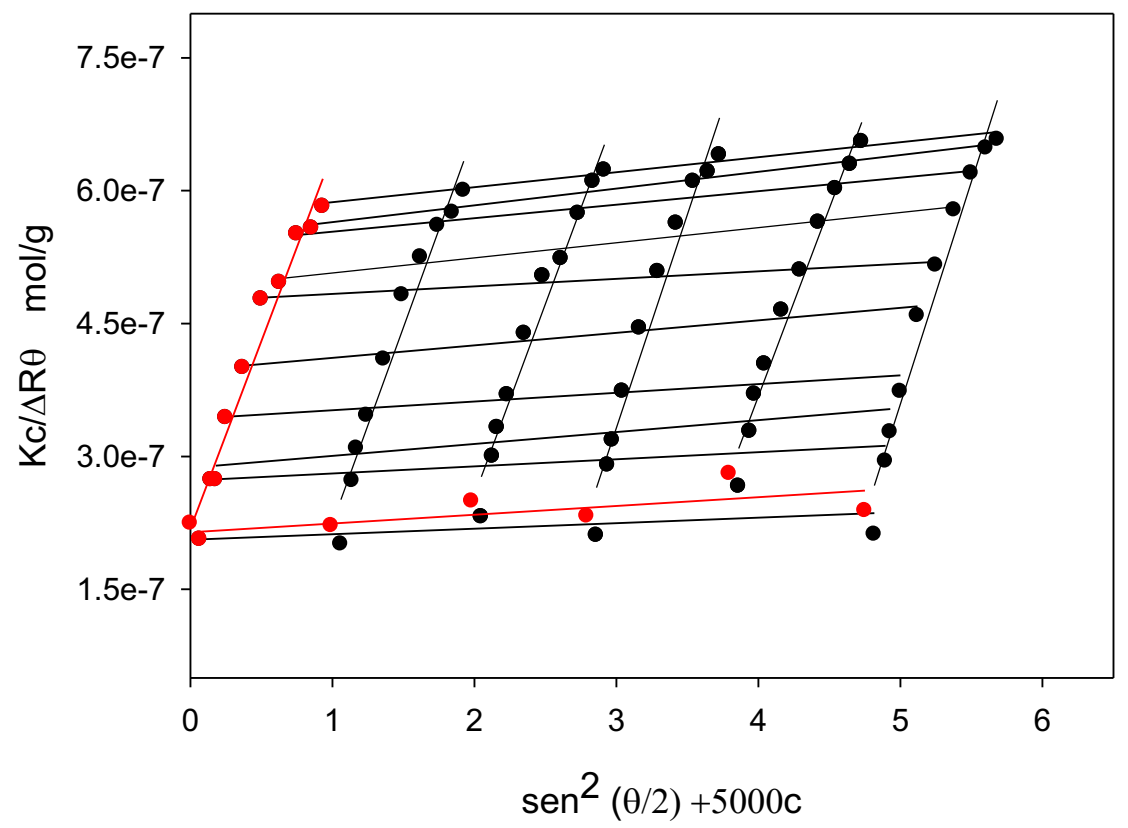

Figure S1. Zimm plot analysis of SLS data for kefiran solutions at various concentrations in the range $0.02-0.2 \% \mathrm{w}$ (red dots represent the extrapolations to concentration $\mathrm{c}=0$ and angle $\theta=0$ ). 

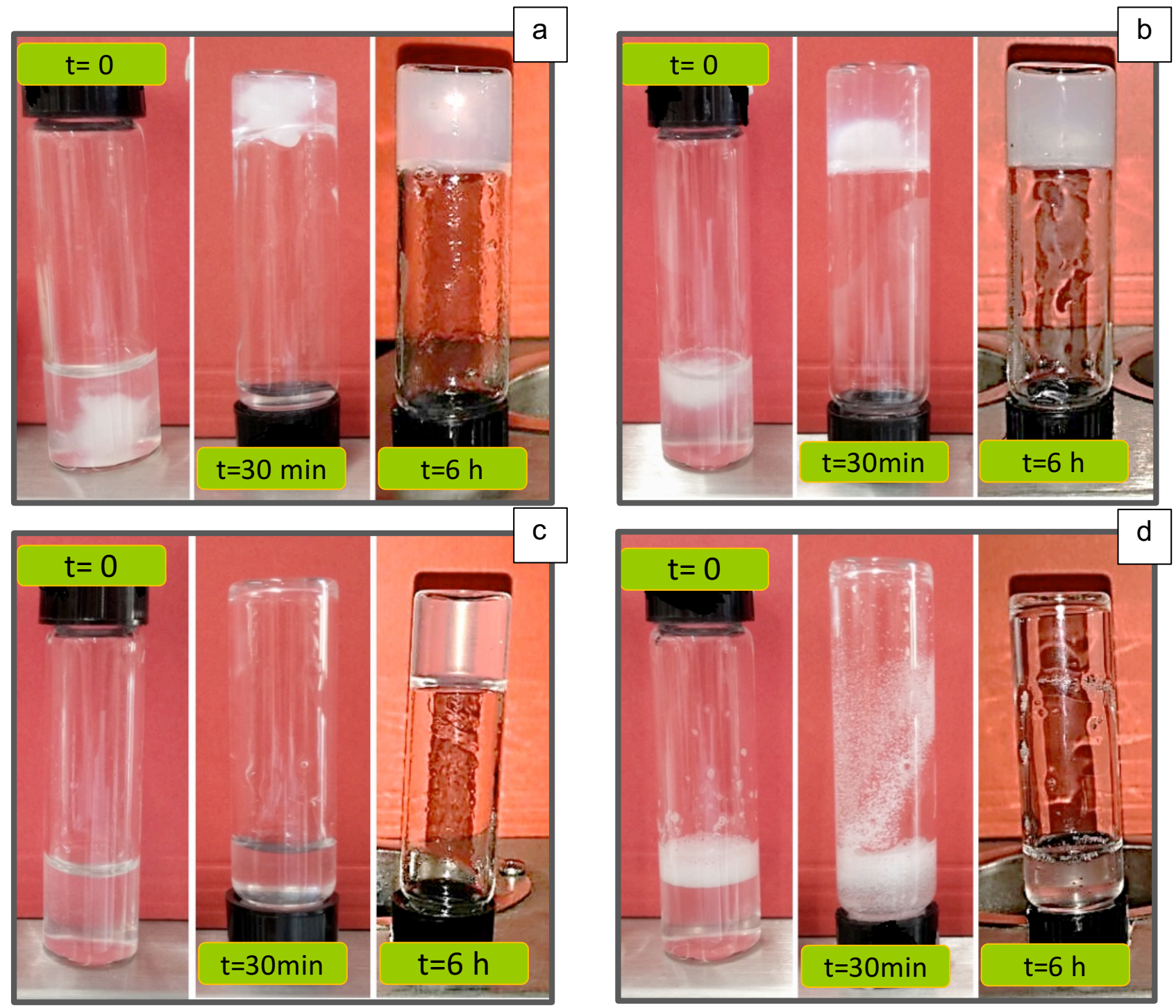

Figure S2. Tilting behavior at room temperature of $\operatorname{Kef}(4)$ solutions immediately after addition of the alcohol ( $\mathrm{t}=0$ ), after 30 minutes and after 6 hours: (a) EtOH; (b) EEE; (c) PG; (d) Gro. 

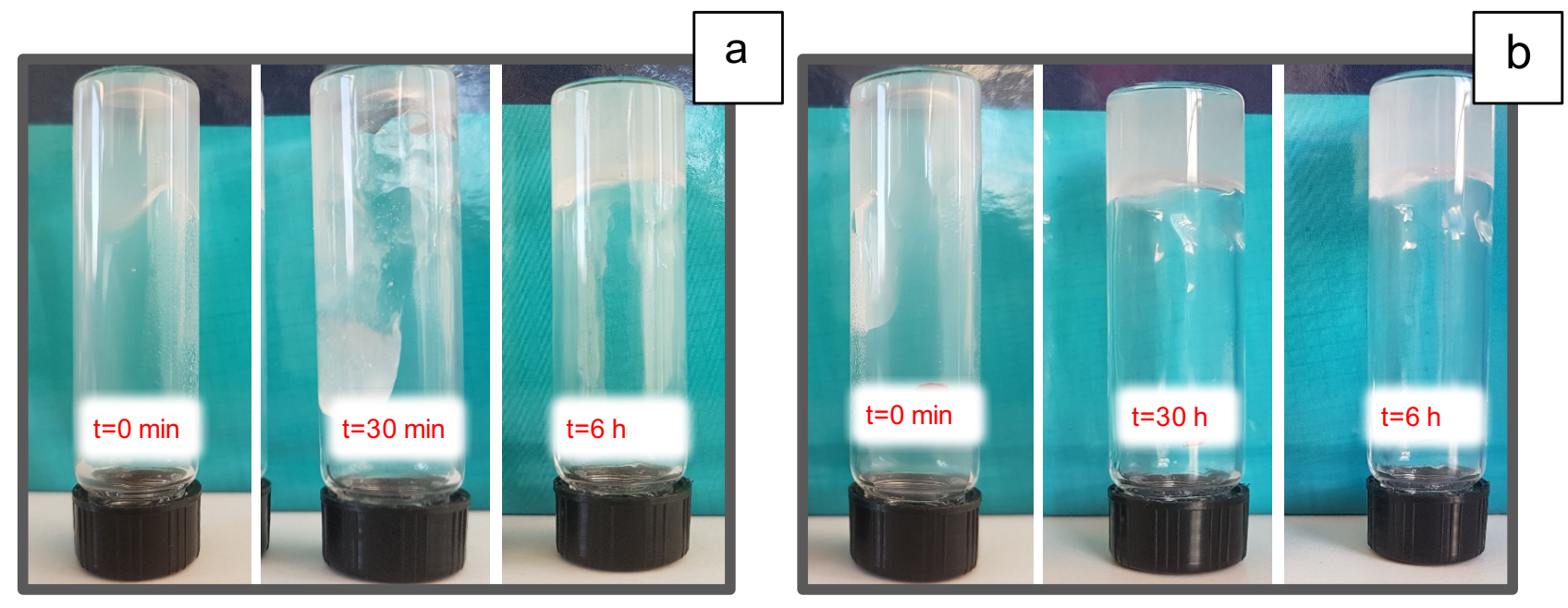

Figure S3 Tilting behavior at room temperature of kefiran solutions immediately after addition of PG ( $\mathrm{t}=0$ ), after 30 minutes and after 6 hours: (a) $\operatorname{Kef}(5)$; (b) $\operatorname{Kef}(6)$. 

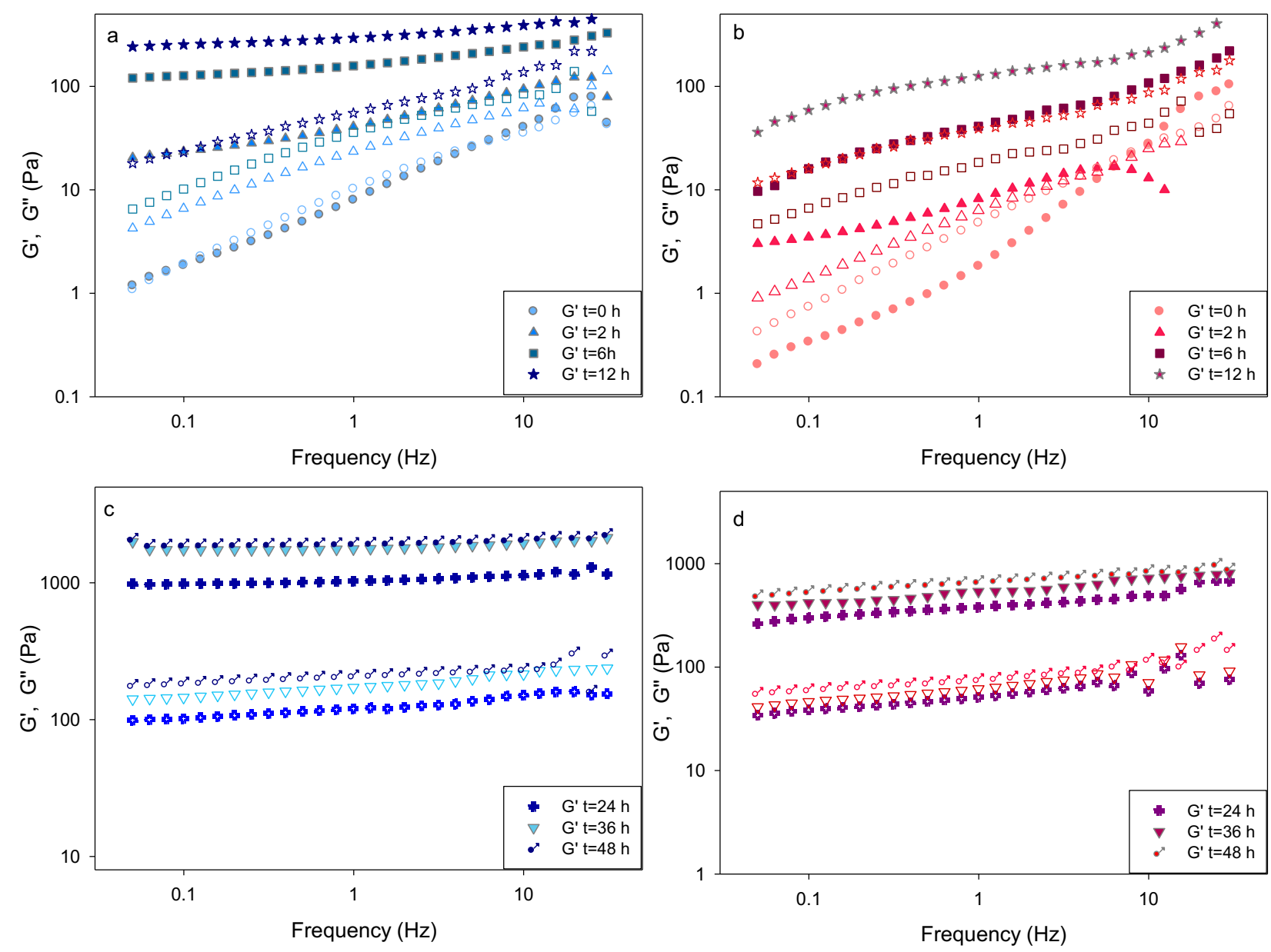

Figure S4. G' and G', as a function of the frequency for Kef (6)/PG stored at $25^{\circ} \mathrm{C}$ for $1,2,6,12 \mathrm{~h}$ (a); at $37^{\circ} \mathrm{C}$ for $1,2,6,12 \mathrm{~h}(\mathrm{~b})$; at $25^{\circ} \mathrm{C}$ for 24,36 and $48 \mathrm{~h}(\mathrm{c})$; at $37^{\circ} \mathrm{C}$ for 24,36 and $48 \mathrm{~h}(\mathrm{~d})$. 